\title{
Immunomodulatory potential of anticancer therapy composed of methotrexate nanoconjugate and dendritic cell-based vaccines in murine colon carcinoma
}

\author{
AGNIESZKA SZCZYGIEŁ, NATALIA ANGER-GÓRA, KATARZYNA WĘGIEREK-CIURA, \\ JAGODA MIERZEJEWSKA, JOANNA ROSSOWSKA, TOMASZ M. GOSZCZYŃSKI, \\ MARTA ŚWITALSKA and ELŻBIETA PAJTASZ-PIASECKA
}

Ludwik Hirszfeld Institute of Immunology and Experimental Therapy, Polish Academy of Sciences, 53-114 Wroclaw, Poland

Received August 27, 2020; Accepted November 30, 2020

DOI: $10.3892 /$ or.2021.7930

\begin{abstract}
Chemotherapy with low-molecular weight compounds, despite elimination of cancer cells, entails adverse effects. To overcome this disadvantage, innovative drug delivery systems are being developed, including conjugation of macromolecular carriers with therapeutics, e.g. a nanoconjugate of hydroxyethyl starch and methotrexate (HES-MTX). The purpose of the present study was to determine whether HES-MTX, applied as a chemotherapeutic, is able to modulate the immune response and support the antitumor response generated by dendritic cells (DCs) used subsequently as immunotherapeutic vaccines. Therefore, MTX or HES-MTX was administered, as sole treatment or combined with DC-based vaccines, to MC38 colon carcinoma tumor-bearing mice. Alterations in antitumor immune response were evaluated by multiparameter flow cytometry analyses and functional assays. The results demonstrated that the nanoconjugate possesses greater immunomodulatory potential than MTX as reflected by changes in the landscape of immune cells infiltrating the tumor and increased cytotoxicity of splenic lymphocytes. In contrast to MTX, therapy with HES-MTX as sole treatment or combined with DC-based vaccines, contributed to significant tumor growth inhibition. However, only treatment with HES-MTX and DC-based vaccines activated the systemic specific antitumor response. In conclusion, due to its immunomodulatory properties, the HES-MTX nanoconjugate could become a potent anticancer agent used in both chemo- and chemoimmunotherapeutic treatment schemes.
\end{abstract}

Correspondence to: Mrs. Agnieszka Szczygieł, Ludwik Hirszfeld Institute of Immunology and Experimental Therapy, Polish Academy of Sciences, 12 Rudolf Weigl Street, 53-114 Wroclaw, Poland

E-mail: agnieszka.szczygiel@hirszfeld.pl

Key words: methotrexate, nanoconjugate, dendritic cells, colon carcinoma, MC38, immunotherapy, chemotherapy

\section{Introduction}

For many years, chemotherapy has been one of the most frequently chosen types of anticancer treatment. However, its cure rate still remains unsatisfactory and additionally severe side effects are observed $(1,2)$. Therefore, the development of effective anticancer therapy is still a challenge, mainly due to the complex nature of tumors (3). During cancer growth, a specific niche-the tumor microenvironment (TME)-is created, consisting mainly of proliferating tumor cells, extracellular matrix, stromal cells and infiltrating immune cells (3-6). Among the latter, high influx of regulatory $\mathrm{T}$ cells (Tregs), tumor-associated macrophages (TAMs) and myeloid derived suppressor cells (MDSCs), which promote tumor progression and suppress the antitumor immune response, are observed (5-7). On the other hand,immune cells, such as effector $\mathrm{T}$ cells, natural killer (NK) cells, M1-type macrophages and dendritic cells (DCs) infiltrating the tumor, can be activated in situ in order to inhibit tumor growth and prevent immune evasion and expansion of the disease (5,7). Increasing evidence indicates that the fate of tumor progression is highly correlated with the specific TME, whose composition is a predominant factor in prognosis and efficacy of chemotherapy (3).

DCs as professional antigen-presenting cells are potent inducers of a $\mathrm{T}$ cell response, and are considered an essential component of antitumor immunity (8). However, despite high potential in promoting the antitumor response, the proper function of DCs present in the TME may be impaired, mainly due to the abundance of immunosuppressive factors and aforementioned cells with suppressor activity. DCs under the influence of a hostile tumor milieu become ineffective in their differentiation and activation, and in turn are weak stimulators of immune responses $(8,9)$. For this reason, great efforts are made to design therapeutic strategies able to overcome the negative impact of TME on endogenous DCs. One of the strategies is ex vivo generation and maturation of DCs for their administration as cellular-based antitumor vaccines (10-12). Other strategies involve, not only developing new chemotherapeutics or innovative solutions for targeted drug delivery, but also combining different types of anticancer therapy-for example chemotherapy and immunotherapy with DC-based cellular 
vaccines. This latter strategy has several immune-potentiating effects, such as increasing the susceptibility of tumor cells to the activity of cytotoxic T lymphocytes (CTLs). Furthermore, by depleting certain population of immune cells, e.g. MDSCs or Tregs, chemotherapy creates a cytokine milieu for optimal expansion of effector cells and facilitates the generation of a specific antitumor immune response by DCs (13). However, major problems in anticancer chemotherapy with low-molecular weight compounds are their fast metabolism and excretion from an organism, as well as unfavorable biodistribution and low specificity (14). To overcome these disadvantages, many different drug delivery systems, including micelles $(15,16)$, dendrimers $(17,18)$, nanocapsules (19) and nanoconjugates with a macromolecular carrier $(14,20)$ have been developed. The nanoconjugates were designed to enhance delivery and to improve the selectivity and pharmacological properties of both conventional and innovative drugs (14). One of these innovative formulations is a nanoconjugate of hydroxyethyl starch (HES) and methotrexate (MTX). The HES-MTX nanoconjugate was obtained by covalent coupling of well-known therapeutic compounds-methotrexate as an anticancer agent and hydroxyethyl starch as a high-molecular carrier $(20,21)$. MTX is one of the oldest antifolate drugs widely used in the treatment of autoimmune disorders as well as in anticancer therapy-in solid tumors and hematologic malignancies (22). However, therapy with MTX is often associated with severe systemic toxicity, bone marrow suppression, and drug resistance $(15,23,24)$. HES is an amylopectin-based modified polymer used as colloidal plasma volume expanders (20). The structural similarity of amylopectin to glycogen ensures lack of immunogenicity (25). Moreover, unfavorable accumulation in the liver or spleen was not observed (26). The mean hydrodynamic diameter of the HES-MTX nanoconjugate is $15.2 \pm 6.2 \mathrm{~nm}$, thus HES-MTX meets the criterion for inclusion in nanoparticles (20). The main advantage of the HES-MTX nanoconjugate over MTX in free form is the prolonged half-time in plasma and specific biodistribution. Methotrexate enters cells via folate receptors (FRs) overexpressed on cancer cells or through the ubiquitously expressed reduced folate carriers (RFCs), to which MTX has a low and high affinity, respectively (27). However, when multiple MTX molecules are covalently conjugated to a macromolecular carrier (e.g. HES), transport through RFCs does not occur. This is possible due to acquisition of the polyvalence feature as a consequence of the conjugation process $(17,18,28,29)$. We postulate that the internalization of HES-MTX nanoconjugate in tumors is achieved mainly by its interaction with $\mathrm{FR} \alpha$, or through an enhanced vascular permeability and retention effect (EPR). EPR is related to the capacity of macromolecules larger than $40 \mathrm{kDa}$ (hydrodynamic diameter above $10 \mathrm{~nm}$ ) for selective leakage from tumor vessels and accumulation in tumor tissue $(2,29-32)$. Complete physicochemical characteristics of the novel form of HES-MTX nanoconjugate as well as its antitumor activity in murine P388 leukemia and human MV-4-11 leukemia models have been described (20).

Recently considerable attention has been focused on the immunomodulatory properties of certain chemotherapeutic agents, including MTX $(1,19)$, which can act not only as modulators of immune cell phenotype (33-35), but also through stimulation of effector immune cells and elimination of Tregs from the TME (36-40). Utilization of the HES-MTX nanoconjugate in a murine MC38 colon carcinoma model and supplementing such anticancer therapy with DC-based immunotherapy, as well determination of its immunomodulatory effect on generation of an antitumor immune response, has not been investigated to date. For this reason, the main objective of our study was to determine the modulation of the immune response after HES-MTX administration to tumor-bearing mice and how those changes affect the activity of DC-based vaccines injected after chemotherapy. The gathered data indicate that chemotherapy with HES-MTX applied in treatment of mice with a subcutaneously growing MC38 tumor affected, more strongly than MTX, the TME by increasing the influx of CTLs and NK cells and eliminating certain cells with suppressor activity. In addition, the enlargement of T-helper (Th), CTL and natural killer T (NKT) cell percentages among splenic leukocytes accompanied by a decrease in Tregs was found. Moreover, therapy with HES-MTX resulted in increased cytotoxic activity of splenic lymphocytes. All these factors led to the creation of a favorable niche necessary to promote the development of an efficient antitumor immune response by DCs used in immunotherapy. The combined therapy with HES-MTX and DC-based cellular vaccines contributed to enhanced influx of effector lymphocytes into tumor tissue and reduced infiltration of immune cells with suppressor activity. The above changes together with activation of systemic specific antitumor response resulted in a significant delay in tumor growth.

\section{Materials and methods}

Mice. Female C57BL/6 mice (total number of animals, 55 mice; initial weight, 20-22 g) were obtained from the Center of Experimental Medicine of the Medical University of Białystok (Białystok, Poland). Mice were kept in a room with a standard light/dark cycle, with a constant temperature $\left(22 \pm 2^{\circ} \mathrm{C}\right)$, air humidity $(55 \pm 10 \%)$ and access to food and water ad libitum. All experiments were performed in accordance with EU Directive 2010/63/EU for animal experiments and were approved by the 1st Local Ethics Committee for Experiments with the Use of Laboratory Animals, Wrocław, Poland (authorization no. 31/2016). After the experiments, mice were sacrificed by cervical dislocation.

Cell culture. The in vivo growing MC38 murine colon carcinoma from the Tumor Bank of the TNA Radiobiology Institute (Rijswijk, The Netherlands) was adapted to in vitro conditions as described by Pajtasz-Piasecka et al (41). The culture of MC38/0 (named here MC38) cells was maintained in RPMI-1640 (Gibco; Thermo Fisher Scientific, Inc.) supplemented with $100 \mathrm{U} / \mathrm{ml}$ penicillin, $100 \mathrm{mg} / \mathrm{ml}$ streptomycin, $0.5 \%$ sodium pyruvate, $0.05 \mathrm{mM} 2$-mercaptoethanol and $5 \%$ fetal bovine serum (FBS; all reagents from Sigma-Aldrich; Merck KGaA). Tumor antigen (TAg) was prepared by repeated freezing and thawing of an MC38 cell suspension $\left(5 \times 10^{6}\right.$ MC38 cells $/ \mathrm{ml}$ ), which was followed by sonication. DCs for in vivo experiments were generated from bone marrow isolated from femurs and tibias of healthy C57BL/6 mice according to the protocol described in our previous publication (42). The cells (named here DCs) were cultured in RPMI 
supplemented with $10 \%$ FBS in the presence of recombinant murine (rm)GM-CSF (ImmunoTools, $40 \mathrm{ng} / \mathrm{ml}$ ) and rmIL-4 (ImmunoTools, $10 \mathrm{ng} / \mathrm{ml}$ ). After 6 days the loosely attached immature DCs were stimulated with tumor antigens $(10 \% \mathrm{v} / \mathrm{v})$ and applied to mice as antitumor vaccines.

Nanoconjugate preparation. FITC-HES was synthesized using a modification of methods previously described (43). Briefly, FITC-HES was prepared by the addition of $30 \mathrm{mg}$ of fluorescein isothiocyanate (FITC, isomer I, Sigma-Aldrich; Merck KGaA) dissolved in $5 \mathrm{ml}$ of DMSO to a solution that contained hydroxyethyl starch $(1.2 \mathrm{~g}$ in $20 \mathrm{ml}$ of solution containing $150 \mathrm{mM} \mathrm{NaCl}$ and $50 \mathrm{mM} \mathrm{Na} \mathrm{CO}_{3}$ ). This mixture was stirred for $48 \mathrm{~h}$ at room temperature (RT). Next, the mixture was cooled down to $4^{\circ} \mathrm{C}$ and precipitated with cold acetone $(100 \mathrm{ml})$. The crude product was solubilized in water and dialyzed against ultrapure water for $5 \mathrm{~h}$ at a flow rate of $30 \mathrm{ml} / \mathrm{min}$ (Pellicon XL with Ultracel-10 PLCGC membrane, Millipore). Finally, the conjugate of FITC and HES containing $3.0 \times 10^{-3}$ covalently bound FITC residues per anhydroglucose unit was obtained.

HES-MTX nanoconjugate and FITC-HES-MTX were synthesized using HES 130/0.4 (Voluven, Fresenius Kabi) or FITC-HES and activated MTX (EBEWE Pharma) according to previously described methods $(20,44)$. The following absorption coefficients were used: $8,571 \mathrm{M}^{-1} \mathrm{~cm}^{-1}(372 \mathrm{~nm})$, $70,000 \mathrm{M}^{-1} \mathrm{~cm}^{-1}$ (494 $\mathrm{nm}$ ) for MTX and FITC, respectively. Eventually, the following conjugates were obtained: HES-MTX containing $52 \times 10^{-3}$ covalently bound MTX residues per anhydroglucose unit and FITC-HES-MTX containing $53 \times 10^{-3}$ MTX and $2.9 \times 10^{-3}$ FITC residues per anhydroglucose unit. In this study, the presented MTX concentration referring to the HES-MTX conjugate was based on the total contents of the covalently bound MTX in conjugate. The analysis and characterization of conjugates were performed using a combination of spectrophotometric, chromatographic and light scattering methods based on previously published procedures $(20,45)$. Hydrodynamic parameters of HES and HES-MTX were characterized by dynamic light scattering (DLS). The sample solution was illuminated by a 633 -nm laser, and the light intensity scattered at an angle of $173^{\circ}$ was measured. At least six consecutive measurements of each sample were carried out. All samples were measured at $25^{\circ} \mathrm{C}$ using a Zetasizer Nano ZS (Malvern Instruments, UK) in a $12-\mu 1$ quartz cuvette (size measurement) and folded capillary cells (zeta potential). HES and HES-MTX conjugate concentration was $5.5 \mathrm{mM}$ (AGU). DLS data were analyzed using the dts 6.10 software (Malvern Instruments, UK). The intensity particle size distributions were obtained using the General Purpose algorithm included in the DTS software.

MTT assay. To calculate the half maximal inhibitory concentration $\left(\mathrm{IC}_{50}\right)$ value, MTT assays were performed. The MC38 cells were placed in 96 -well plates $\left(0.005 \times 10^{6}\right.$ cells/well $)$ and after $24 \mathrm{~h}$ MTX or HES-MTX in various concentrations was added (in the range from 0.001 to $1,000 \mathrm{ng} / \mathrm{ml}$ ) and incubated for $72 \mathrm{~h}$. After this time, MTT dye (3-(4,5-dimethylthiazol-2-yl)-2,5-diphenyltetrazolium bromide; $5 \mathrm{mg} / \mathrm{ml}$ ) was added for $4 \mathrm{~h}$. Next, cells were lysed overnight in lysis buffer (N,N-dimethylmethanamide, sodium dodecyl sulfate and water). Absorbance at $570 \mathrm{~nm}$ was determined using a Thermo Labsystems Multiskan RC microplate reader (Thermo Fisher Scientific, Inc.) with Genesis Lite 3.05 Software (Thermo Life Sciences) and the $\mathrm{IC}_{50}$ value was calculated.

Interaction of nanoconjugate with MC38 cells and DCs. The interaction of FITC-conjugated compounds (FITC-HES-MTX and FITC-HES) with MC38 cells and DCs was evaluated by flow cytometry. The MC38 cells were placed in 24-well plates $\left(0.2 \times 10^{6}\right.$ cells/well $)$, immature DCs were placed in 12 -well plates $\left(0.5 \times 10^{6}\right.$ cells/well $)$ and after $24 \mathrm{~h}$ FITC-HES-MTX or FITC-HES $(10 \mu \mathrm{g} / \mathrm{ml})$ was added and incubated for the next $24 \mathrm{~h}$. After this time, cells were harvested and washed, and dead cells were stained with DAPI dye. The analysis was performed using FACS Fortessa with Diva software (Becton Dickinson).

Determination of FR $\alpha$ expression. The expression of FR $\alpha$ was measured by real-time PCR. Total RNA was isolated using a NucleoSpin RNA kit (Macherey-Nagel) and reverse-transcribed with a First Strand cDNA Synthesis Kit (Thermo Fisher Scientific, Inc.). Real-time PCR was performed using TaqMan Universal PCR Master Mix and TaqMan Gene Expression Assay primers for FR $\alpha$ in reference to the HPRT gene. The analyses were performed using the ViiA7 Real-Time PCR System (Applied Biosystem).

Surface plasmon resonance spectroscopy. Surface plasmon resonance (SPR) experiments were conducted in a Biacore T200 instrument (GE Healthcare Life Sciences). During measurements, the flow buffer HBS-N was used $(10 \mathrm{mM}$ HEPES, pH 7.4 with $150 \mathrm{mM} \mathrm{NaCl})$. Immobilization of bovine folate binding protein (FBP, Sigma-Aldrich) was carried out at $25^{\circ} \mathrm{C}$ by an EDC-based amide coupling method using standard Biacore reagents (GE Healthcare Life Sciences). FBP-presenting chips (CM5) were prepared with protein density at $9.03 \mathrm{FBP} \mathrm{ng} / \mathrm{mm}^{2}$. SPR experiments were performed by injection of a ligand solution, HES or HES-MTX nanoconjugate, each prepared in HBS-N buffer (concentrations were presented as MTX-equiv), at a flow rate of $40 \mu \mathrm{l} / \mathrm{min}$. The conjugates were injected over a reference channel and over a channel with immobilized FBP for $200 \mathrm{sec}$. Each analysis cycle consisted of a $60 \mathrm{sec}$ initial period, in which the stability of the baseline was monitored. The injection of buffer was performed between each analysis cycle for a double reference. At the end of each dissociation phase, the chip surface was treated with $10 \mu \mathrm{l}$ of $10 \mathrm{mM}$ glycine- $\mathrm{HCl}(\mathrm{pH} \mathrm{2.5)}$ for surface regeneration. Sensorgrams for the reference channel were subtracted from sensorgrams for the channel with FBP. Subsequently, sensorgrams of buffer were subtracted from sensorgrams of the HES-MTX conjugates.

Annexin $V$ binding assay. To evaluate apoptosis in MC38 cells after $72 \mathrm{~h}$ incubation with MTX or HES-MTX the Annexin V binding assay was performed. Briefly, the MC38 cells were placed in 24 -well plates $\left(0.1 \times 10^{6}\right.$ cells/well $)$ and after $24 \mathrm{~h}$ MTX or HES-MTX was added (500 ng/ml). Next, harvested cells were suspended in binding buffer and stained with Annexin V protein conjugated with APC fluorochrome (Thermo Fisher Scientific, Inc.) (15 min, RT). To determine the 
percentage of dead cells, propidium iodide (PI) was applied (10 $\mu \mathrm{g} / \mathrm{ml}$, Thermo Fisher Scientific, Inc.) and the percentage of Annexin $\mathrm{V}^{+}$MC38 cells was analyzed using FACSCalibur with CellQuest 3.3 Software (Becton Dickinson).

Modulation of maturation and phenotype of DCs generated in the presence of metabolites released by MC38 cells after MTX or HES-MTX treatment. The conditioned medium (CM) necessary to assess modulation of the DC phenotype generated in the presence of metabolites of tumor cells treated with MTX or HES-MTX was freshly prepared before each test. For this purpose, the MC38 cells were placed in 6-well plates $\left(1.15 \times 10^{6}\right.$ cells/well) and $24 \mathrm{~h}$ later MTX or HES-MTX was added (500 ng/ml). Additionally, culture medium containing MTX or HES-MTX without any cells was also prepared. After $72 \mathrm{~h}$ of incubation, CM from the treated MC38 cells was collected, cellular debris was removed by centrifugation $(15 \mathrm{~min}, 2,000 \times \mathrm{g})$ and the obtained CM was used in differentiating culture of DCs from bone marrow. Medium containing MTX or HES-MTX maintained without cells was prepared according to the same procedure. Bone marrow cells $\left(0.5 \times 10^{6}\right.$ cells/well, 12 -well plates $)$ were suspended in a mixture of culture medium (including cytokines necessary for DC generation) and conditioned medium (or medium with MTX or HES-MTX) in a 50:50 ratio. After the first $48 \mathrm{~h}$ of DC generation, the mixture of culture medium and CM or medium containing MTX or HES-MTX without cells was replaced with RPMI supplemented with $10 \% \mathrm{FBS}, 40 \mathrm{ng} / \mathrm{ml}$ rmGM-CSF and $10 \mathrm{ng} / \mathrm{ml} \mathrm{rmIL}-4$. Further DC culture was conducted according to the protocol in our previous publication (42). After 6 days, the loosely attached immature DCs were collected and then stimulated for $24 \mathrm{~h}$ with tumor antigens as described above. The phenotype of mature DCs was analyzed. For this purpose, DCs were stained with anti-CD11c BV650, anti-MHC II FITC, anti-CD40 PE, anti-CD80 APC (all from BioLegend) and anti-CD11c BV650 (BioLegend) with anti-CD86 PE (BD Biosciences). The analysis was performed using FACS Fortessa with Diva software (Becton Dickinson).

Therapeutic treatment schedule. Eight-to 10-week old female C57BL/6 mice (45 mice) were subcutaneously (s.c.) inoculated in the right flank with MC38 cells $\left(1.1 \times 10^{6}\right.$ cells $/ 0.2 \mathrm{ml}$ $\mathrm{NaCl} 0.9 \% /$ mouse). The mice were treated according to the schemes presented in Figs. 3A and 5A. In the course of the chemotherapeutic treatment scheme (results presented in Figs. 3 and 4), on the 14th day of the experiment, mice received intravenously (in tail vein, intravenously; i.v.) MTX or HES-MTX (20 mg/kg body weight) and three days later (17th day of experiment) 5 mice from each group were sacrificed and tumor nodules and spleens were dissected, homogenized and stored in liquid nitrogen for further analyses. In the chemoimmunotherapeutic treatment scheme (results presented in Figs. 5 and 6) mice received chemotherapy on the 14th day of the experiment and on the 17, 24th and 31st day of the experiment tumor antigen-stimulated DC-based vaccines were applied peritumorally (p.t.) (DC/TAg, $2 \times 10^{6}$ cells $/ 0.2 \mathrm{ml} \mathrm{NaCl}$ $0.9 \% / \mathrm{mouse} / \mathrm{p}$.t.injection).In the group of mice from non-treated and chemotherapy-receiving groups (MC38 control, MTX and HES-MTX) tumor nodules and spleens were dissected on the 31st day of the experiment, and from DC/TAg-receiving groups (DC/TAg, MTX+DC/TAg, HES-MTX+DC/TAg) tumor nodules and spleens were dissected on the 35 th day of the experiment (3-5 mice per group). Then tumors and spleens were homogenized and stored in liquid nitrogen for further analyses. The health of the mice was monitored during the experiments (weight loss, bristling hair, lethargy) and tumors were measured by using a calliper two times a week. Mice were sacrificed when the tumor volume was $>2 \mathrm{~cm}^{3}$. The procedure of tumor growth monitoring was presented by Rossowska et al (42). The therapeutic effect of the treatment was evaluated using tumor growth inhibition (TGI), calculated according to the formula: $T G I[\%]=100-\left(T V / T V_{n t} \times 100\right)$, where $T V_{t}$ is the median tumor volume in the treated group of mice and $T V_{n t}$ is the median tumor volume in the non-treated group of mice.

Analysis of myeloid cells and lymphocytes in tumors and spleens of mice after the therapy. Tumor cells and spleen cells isolated from mice were thawed and stained for identification of myeloid or lymphoid cell subpopulations according to the procedure described previously (46). Briefly, tumor single-cell suspensions were stained with the LIVE/DEAD Fixable Violet Dead Staining Kit (Thermo Fisher Scientific, Inc.) and then labelled with cocktails of fluorochrome-conjugated monoclonal antibodies: anti-CD3 PE-CF594, anti-CD19 PE-CF594, anti-CD49b PE-CF594 (all from BD Biosciences), anti-CD45 BV605, anti-CD11b PerCP-Cy5.5, anti-CD11c BV650, anti-F4/80 Alexa Fluor 700, anti-Ly6C PE, anti-Ly6G APC-Cy7, anti-MHC II FITC, anti-CD80 PE-Cy7 (all from BioLegend) for myeloid cell identification, and anti-CD45 BV605, anti-CD3 BV650, anti-CD4 FITC, anti-CD8 APC/Fire 750, anti-CD25 PE (all from BioLegend) for lymphocyte identification. Then, the cells were fixed using the Foxp3/Transcription Factor Staining Buffer Set (eBioscience). Cells stained with myeloid or lymphocyte cocktail were additionally incubated with anti-CD206 APC (BioLegend) or anti-FoxP3 APC (eBioscience) antibodies, respectively. In spleen single cell suspension only the lymphocyte identification was performed according to the procedure described above. The analysis was performed using a FACS Fortessa flow cytometer with Diva software (Becton Dickinson).

Analysis of antitumor response of effector spleen cells. Spleen cells obtained from non-treated or treated tumor-bearing mice were cocultured with mitomycin C-treated MC38 cells (50 mg mitomycin $\mathrm{C} / 3 \times 10^{6}$ cells, $30 \mathrm{~min}$., $37^{\circ} \mathrm{C}$ ) in the presence of recombinant human IL-2 $(200 \mathrm{U} / \mathrm{ml})$. After 5 days of restimulation, supernatants were collected and stored at $4^{\circ} \mathrm{C}$ until ELISA was performed. Cytotoxic activity of cells stained with DiO lipophilic dye (Molecular Probes) was analyzed according to a previously described procedure (47). Two E:T (effector to target) ratios were investigated: 10:1 and 30:1. The percentage of dead double positive $\left(\mathrm{DiO}^{+} \mathrm{PI}^{+}\right) \mathrm{MC} 38$ cells was determined after analysis using a FACSCalibur with CellQuest 3.3 software (Becton Dickinson). In order to determine the percentage of $\mathrm{CD} 107 \mathrm{a}^{+}$cells, restimulated spleen cells were incubated for $2 \mathrm{~h}$ with MC38 cells in the presence of monoclonal anti-CD107a antibody conjugated with APC (BioLegend). Afterwards, cells were stained with anti-CD45 V500, anti-CD4 FITC, anti-CD8 PE-Cy7 and anti-CD49b 

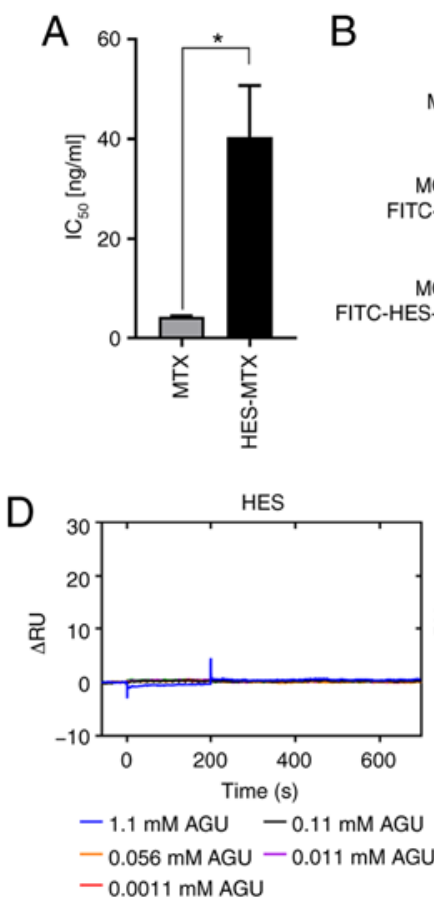

B

B
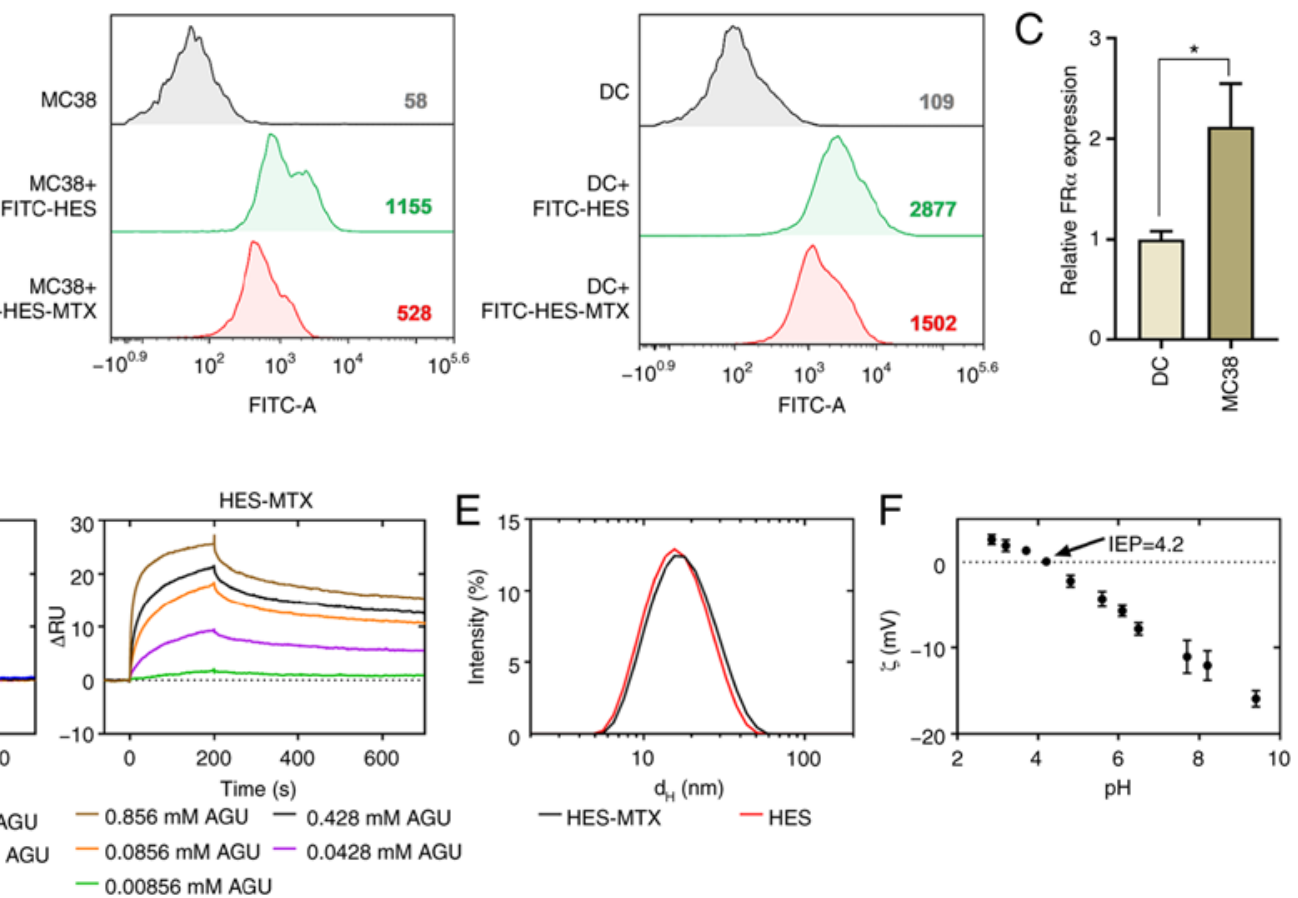

Figure 1. (A) Antiproliferative activity of MTX and HES-MTX against MC38 cells after a 72-h treatment was measured by MTT assay and the IC S0 $_{50}$ value was calculated. (B) Representative histogram showing the interaction of FITC-conjugated HES-MTX and HES with MC38 cells and DCs after a 24-h treatment measured by flow cytometry. The numbers presented on the histograms represent mean fluorescence intensity (MFI) values. (C) Relative expression of folate receptor $\alpha(\mathrm{FR} \alpha)$ measured by real-time PCR in murine DCs and MC38 cells. (D) Representative sensorgrams from SPR measurements showing the association and dissociation phase of HES and HES-MTX conjugate from FBP immobilized on the surface of the CM5 chip. Sensorgrams are shown after subtraction for reference channel and blank measurements. Concentrations for both HES and HES-MTX are shown as anhydroglucose unit (AGU). (E) Size distributions are shown according to intensity measured by dynamic light scattering technique. $\mathrm{d}_{\mathrm{H}}$, mean hydrodynamic diameter. $(\mathrm{F})$ Zeta $(\zeta)$ potential of HES-MTX as a function of $\mathrm{pH}$ measured by dynamic light scattering technique. The points denote experimental values determined for ionic strength $0.15 \mathrm{mM}$; IEP, isoelectric point. Results $(\mathrm{A}$ and $\mathrm{C})$ are expressed as the mean $\pm \mathrm{SD}$ calculated for at least three independent experiments. Differences between the groups were estimated by (A) Mann-Whitney test ( $\left.{ }^{*} \mathrm{P}<0.05\right)$ and $(\mathrm{C})$ unpaired $\mathrm{t}$-test ( $\left.\mathrm{P}<0.05\right)$. HES, hydroxyethyl starch; MTX, methotrexate; DC, dendritic cells.

PE and analyzed using a FACS Fortessa with Diva software (Becton Dickinson).

Determination of cytokine production. Production of cytokines by restimulated spleen cells was evaluated using commercially available ELISA kits (IL-10, IL-4; BD Biosciences and IFN- $\gamma$; eBioscience) according to the manufacturer's instructions.

Statistics. All the data were analyzed using GraphPad Prism 8 software (GraphPad Software, Inc.). The normality of residuals was confirmed by the D'Agostino-Pearson omnibus test. When data were consistent with a Gaussian distribution and had equal SD values, the statistical significance was calculated using the parametric one-way ANOVA followed by Tukey's multiple comparison post-hoc test. When data were consistent with a Gaussian distribution but SD values were not equal, the Brown-Forsythe and Welch ANOVA test followed by Dunnett's T3 multiple comparisons post-hoc test was performed. Data inconsistent with a Gaussian distribution were analyzed using the nonparametric Kruskal-Wallis test for multiple independent groups followed by Dunn's multiple comparison post-hoc test. In analyses where only two groups were compared, the statistical differences were calculated using the Mann-Whitney test or unpaired t-test. The statistical significance in kinetics of tumor growth was calculated using the two-way ANOVA followed by Bonferroni's multiple comparisons post-hoc test. The type of statistical analysis used is described in the captions under the figures. All statistically significant differences are presented in the graphs; otherwise the differences were not significant.

\section{Results}

Antiproliferative activity of nanoconjugate against MC38 cells in vitro. The first step of our research was to determine in vitro activity of the HES-MTX nanoconjugate against MC38 cells. Therefore, its antiproliferative activity was evaluated using the MTT assay (Fig. 1A). Based on the calculated $\mathrm{IC}_{50}$ value, the HES-MTX nanoconjugate demonstrated a 10 -fold weaker antiproliferative effect against MC38 cells than MTX. In order to confirm the interaction of HES-MTX with MC38 cells, FITC dye was attached to the HES molecule and analysis by flow cytometry revealed that despite its larger diameter, HES-MTX appeared to be able to enter tumor cells (Fig. 1B). To evaluate the interaction of HES-MTX with DCs, the same analysis was performed. In comparison to the MC38 cells, DCs interacted more strongly with HES and HES-MTX, which was reflected in higher mean fluorescence intensity (MFI) values. However, this phenomenon may be related to the increased uptake capacity of these cells when compared to tumor cells. Taking into account the polyvalence of HES-MTX, the nanoconjugate could interact with cells through FR $\alpha$, which is abundant on cancer cells. Determination of the expression of FR $\alpha$ in MC38 cells revealed that in comparison to DCs, 
MC38 cells showed significantly higher expression of FR $\alpha$ (Fig. 1C). The affinity of the HES and the HES-MTX nanoconjugate to folate binding protein (FBP) was investigated using SPR. HES (a negative control without MTX molecules attached) did not show any significant response over the studied concentration range (1.1-0.0011 mM AGU), indicating a lack of affinity to the FBP surface (Fig. 1D). In contrast, the HES-MTX showed an increasing concentration-dependent response. The shape of the sensorgrams indicated a fast and strong association of the HES-MTX on the FBP presenting surface. Dissociation curves for HES-MTX indicated that the conjugate dissociates with complex kinetics, initially at a fast and subsequently at slower rates. At the end of analysis, the dissociation phase was still incomplete. This suggests a high affinity of the nanoconjugate to FBP. Characterization of HES and HES-MTX by light scattering technique revealed that HES-MTX represents a typical batch of HES polymers with a mean hydrodynamic diameter of about $15 \mathrm{~nm}$ when compared to the initial (unmodified) polymer ( 14 nm) (Fig. 1E). The surface of HES-MTX nanoconjugate has a negative zeta $\zeta$ potential [about-10 $\mathrm{mV}$ at $\mathrm{pH}=7.4$, isoelectric point $($ IEP $)=4.2$ ] (Fig. 1F). The gathered data demonstrated that the HES-MTX nanoconjugate has weaker antiproliferative activity against tumor cells than the free form of MTX. Moreover, HES after conjugation with MTX gains a high affinity to FBP and is a good candidate as a folate targeting macromolecule (an FR-targeted chemotherapeutic). We assume that, due to the acquired properties of HES-MTX, it should act more specifically towards tumor cells and have greater potential as a chemotherapeutic agent.

Modulation of maturation and phenotype of DCs generated in the presence of metabolites released by MC38 cells after MTX or HES-MTX treatment. It was found that certain chemotherapeutics, including MTX, used in appropriate concentrations, can act as immunomodulators and contribute to an increase in DC maturation $(33,34)$. To investigate the impact of the nanoconjugate on DC generation and phenotypic changes as well as to assess whether the nanoconjugate could reverse the inhibitory effect of MC38 cells on maturation of DCs, in vitro studies were conducted. For this purpose, the percentage of Annexin $\mathrm{V}^{+}$MC38 cells previously treated with MTX or HES-MTX was determined (Fig. 2A). Similar to the observation made in the MTT assay and calculated $\mathrm{IC}_{50}$ value, the Annexin V binding assay revealed that the HES-MTX nanoconjugate was less effective in induction of apoptosis than MTX. To reflect the changes in the TME occurring after nanoconjugate treatment and the potential effect on DC maturation, the MC38 cells were treated with MTX or HES-MTX for $72 \mathrm{~h}$. Subsequently, the conditioned medium (CM) harvested from treated MC38 cells was used in ex vivo generation of $\mathrm{DCs}$ (named hereafter as DC/MC38/TAg, DC/MC38/MTX/TAg and DC/MC38/HES-MTX/TAg). Due to the immunomodulatory effect of the nanoconjugate and MTX on DC phenotype, the culture medium containing MTX or HES-MTX without any cells was incubated for $72 \mathrm{~h}$ and then used in in vitro studies (named hereafter as DC/MTX/TAg and DC/HES-MTX/TAg). Next, in order to obtain the mature DCs, immature DCs were stimulated with tumor antigens (TAg) and phenotype alterations of mature DCs were evaluated by flow cytometry.
The phenotype analysis of mature DCs showed that in comparison to the non-treated DCs (DC/TAg group), the presence of tumor metabolites (DC/MC38/TAg) did not influence

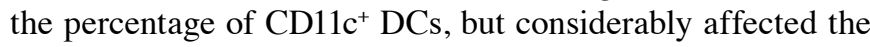
maturation of these cells (Fig. 2B and C). DCs generated in CM harvested from MC38 cells (DC/MC38/TAg) were characterized by statistically significantly lower expression of MHCII and CD40, CD80 and CD86 co-stimulatory molecules compared to the non-treated DC/TAg group. However, this effect was partially restored when MTX or HES-MTX was used. When CM from above MC38 cells treated with MTX or HES-MTX was used in generation of DCs (DC/MC38/MTX/TAg and DC/MC38/HES-MTX/TAg), those cells were characterized by a statistically significantly lower percentage of $\mathrm{CD}_{11 \mathrm{c}^{+} \text {cells }}$ compared to DC/TAg and DC/MC38/TAg (Fig. 2B). When we applied medium from the above MC38 cells treated with MTX (DC/MC38/MTX/TAg), expression of the analyzed antigens (Fig. 2C) was significantly higher than that observed after using HES-MTX (DC/MC38/HES-MTX/TAg). We postulate that this effect was related to the lower antiproliferative activity of HES-MTX than MTX against tumor cells. In comparison to MTX (DC/MTX/TAg), the nanoconjugate itself has an immunomodulatory effect on the phenotype of DCs (DC/HES-MTX/TAg), which was observed especially with a statistically significantly greater percentage of $\mathrm{CD} 11 \mathrm{c}^{+}$ cells and higher expression of co-stimulatory molecules.

Summarizing these results, in comparison to the nanoconjugate, the treatment of MC38 cells with MTX (DC/MC38/MTX/TAg) resulted in more efficient abolition of the negative effect of tumor cells on DC generation and maturation. However, when considering the influence of MTX or HES-MTX on the DC phenotype and their response to stimulation with TAg, the nanoconjugate (DC/HES-MTX/TAg) was a more efficient immunomodulator of DC maturation.

Influence of nanoconjugate administration on activation of local and systemic antitumor response in the MC38-bearing mice. To answer the question whether therapy with the novel MTX conjugate modulates the local and systemic antitumor response and how it would affect the efficacy of DC-based vaccines administered after chemotherapy, in vivo experiments were conducted. In our previous chemoimmunotherapy schedules in the MC38-tumor model, DC-based vaccines were applied three days after cyclophosphamide administration $(39,40,46)$. Therefore, in the present experiment, we aimed to determine what changes in the tumor and spleen would occur as a result of the application of MTX or the nanoconjugate HES-MTX. For this purpose, mice with subcutaneously growing MC38 tumor received MTX or HES-MTX i.v. and three days later the tumor nodules and spleens were dissected for further analyses (Fig. 3A). Flow cytometric analyses allowing the simultaneous identification of multiple immune cell subpopulations in tumor and spleen tissues were performed (Fig. 3B for tumors and Fig. 4A for spleens).

In the tumor tissue, we examined the percentage of $\mathrm{CD}_{4} 5^{+}$ cells (leukocytes) and among them we identified myeloid cells, TAMs $\left(\mathrm{CD} 11 \mathrm{~b}^{+} \mathrm{CD} 11 \mathrm{c}^{+} \mathrm{F} 4 / 80^{+}\right)$, macrophages (Mfs)

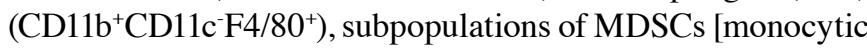
(M-)MDSCs (CD11b ${ }^{+}$CD11c-F4/80-'-Ly6C ${ }^{+} \mathrm{Ly}^{-} \mathrm{G}^{-}$), polymorphonuclear (PMN-)MDSCs (CD11b+CD11c-F4/80-Ly6C ${ }^{\text {int }}$ Ly6G $\left.^{+}\right)$], 

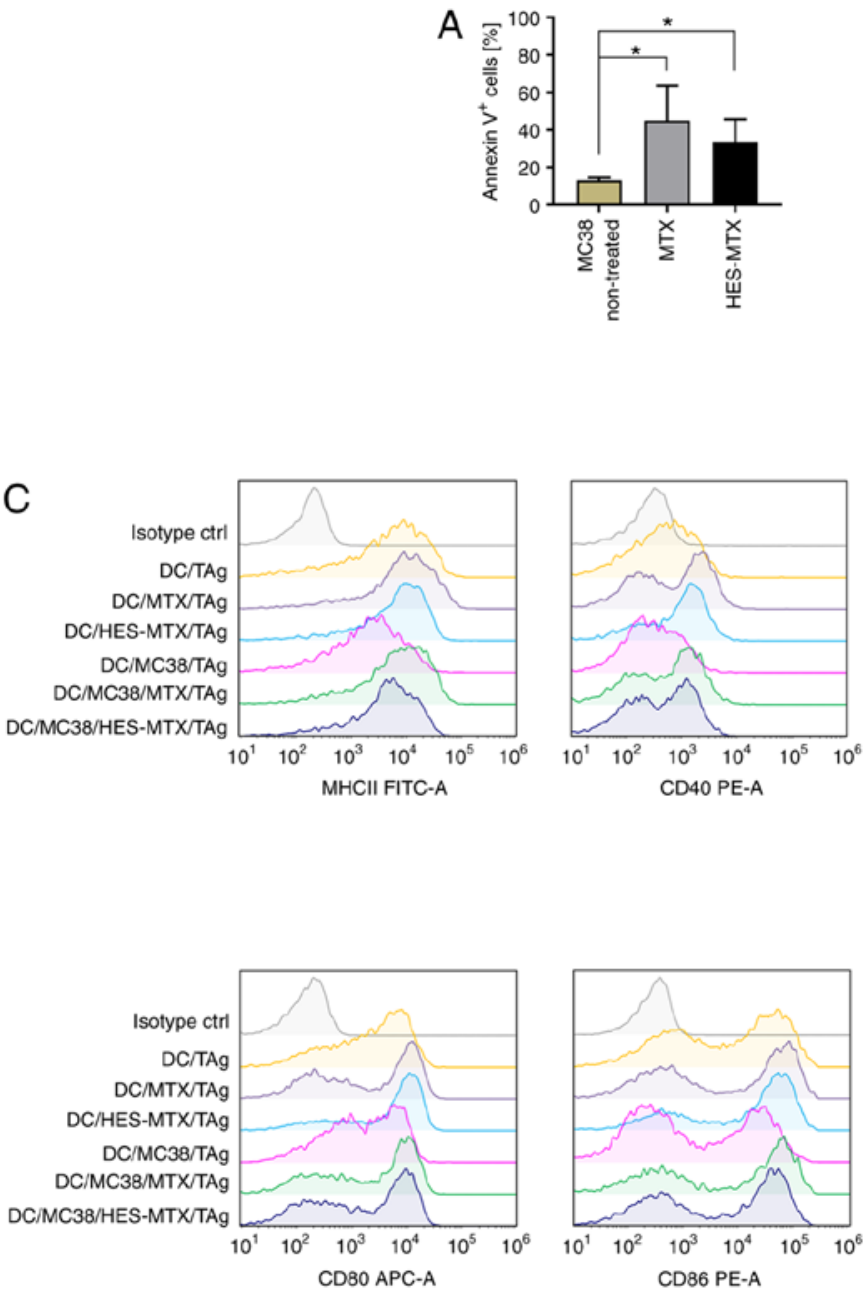
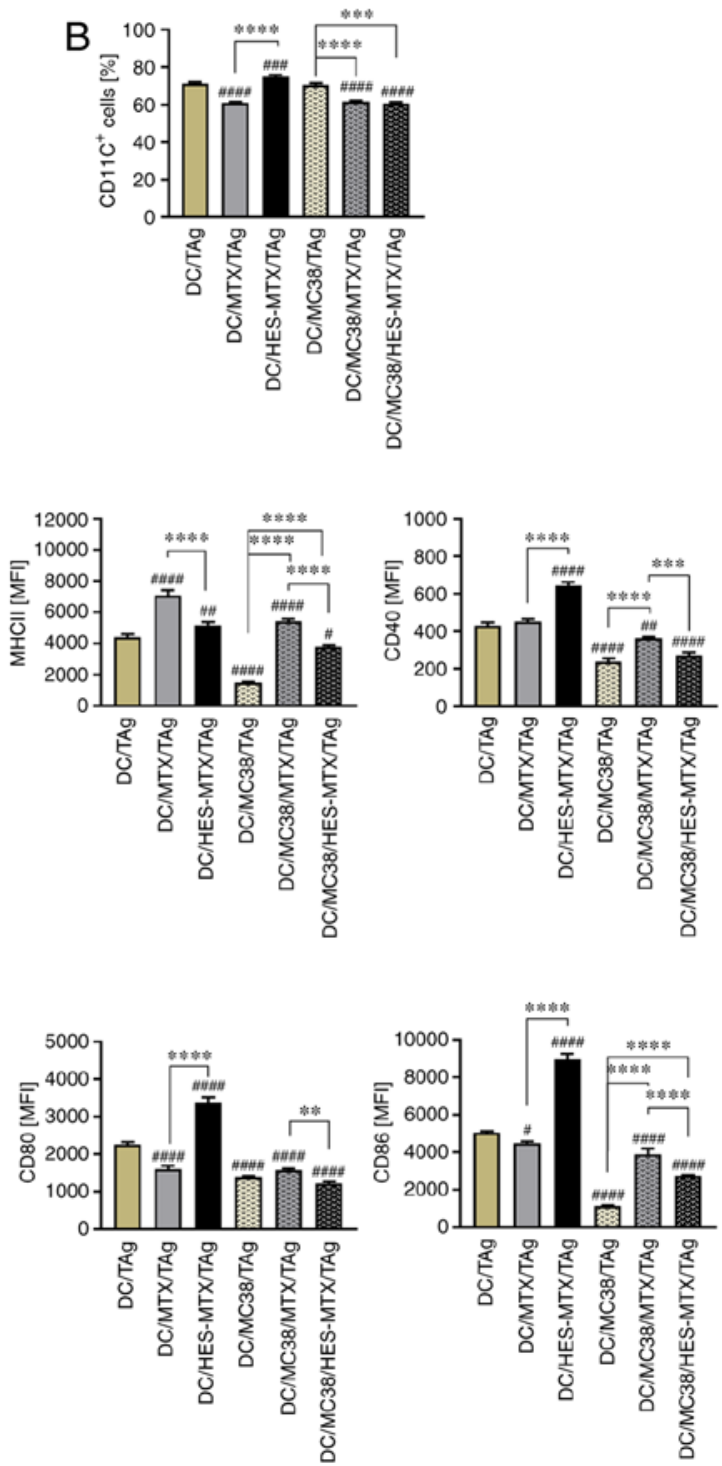

Figure 2. Modulation phenotype of mature DCs generated in the presence of metabolites released by MC38 cells after MTX or HES-MTX treatment. (A) Percentage of Annexin $\mathrm{V}^{+}$MC38 cells after a 72-h treatment with MTX or HES-MTX as determined by Annexin V binding assay; (B) Bar plots showing the percentage of CD11 $\mathrm{c}^{+}$mature DCs (stimulated with TAg) (C) Bar plots and representative histograms showing the expression of MHC II and costimulatory molecules on mature DCs. Results are expressed as mean \pm SD calculated for three independent experiments. Differences between groups were calculated using (A) the Brown-Forsythe and Welch ANOVA test followed by Dunnett's T3 multiple comparisons post-hoc test or (B and C) one-way ANOVA followed by Tukey's multiple comparison post-hoc test. The asterisks ${ }^{*}$ ) presented in the graphs indicate statistically significant differences between the given groups; a hashtag $\left(^{\#}\right)$ above a bar indicates a statistically significant difference between the given group and the control group-DC/TAg $\left({ }^{* / \# /} \mathrm{P}<0.05,{ }^{* * / \# / \#} \mathrm{P}<0.01\right.$,

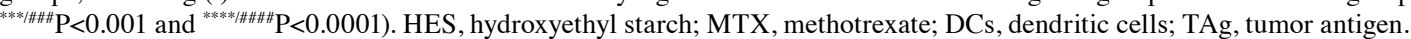

DCs $\left(\mathrm{CD} 11 \mathrm{~b}^{+} \mathrm{CD} 11 \mathrm{c}^{+} \mathrm{F} 4 / 80^{\text {int }} \mathrm{MHCII}^{+}\right)$, and expression of MHC II and CD80 molecules on the surface of DCs. Moreover, polarization of macrophages toward type M1 $\left(\mathrm{CD} 11 \mathrm{~b}^{+} \mathrm{F} 4 / 80^{+} \mathrm{CD} 206\right)$ and $\mathrm{M} 2\left(\mathrm{CD} 11 \mathrm{~b}^{+} \mathrm{F} 4 / 80^{+} \mathrm{CD} 206^{+}\right)$was identified by evaluation of CD206 intracellular antigen expression. Then the M1/M2 ratio was calculated. In addition, among $\mathrm{CD} 45^{+}$cells in tumor tissues we identified lymphoid cells, $\mathrm{CD}^{+}{ }^{+}\left(\mathrm{CD}^{+}{ }^{+} \mathrm{CD} 4^{+}\right)$, Tregs $\left(\mathrm{CD}^{+} \mathrm{CD}^{+}{ }^{+} \mathrm{CD} 25^{+} \mathrm{FoxP}^{+}\right), \mathrm{CD}^{+}{ }^{+}$ $\left(\mathrm{CD}^{+}{ }^{+} \mathrm{CD} 8^{+}\right), \mathrm{NK}\left(\mathrm{CD} 49 \mathrm{~b}^{+}\right)$and $\mathrm{NKT}\left(\mathrm{CD}^{+}{ }^{+} \mathrm{CD} 49 \mathrm{~b}^{+}\right)$cells.

After application of MTX or HES-MTX, a high influx of $\mathrm{CD}_{4} 5^{+}$cells in tumor tissue was observed (Fig. 3C). In both MTX and HES-MTX groups, a lower percentage of TAMs and Mfs was found (Fig. 3D and E); however, none of these changes was statistically significant. Although this effect was accompanied by high infiltration of M-MDSCs, the percentage of
PMN-MDSCs remained at the same, low level (Fig. 3F and G). It should be highlighted, that after HES-MTX application we noted the lowest influx into tumor tissue of TAMs, which presumably belong to M1 type macrophages. The M1/M2 ratio was not significantly elevated only after application of HES-MTX, which would suggest a potent impact of the nanoconjugate on the level of macrophage polarization toward M1 type (Fig. 3H). It should be highlighted, that neither MTX nor HES-MTX elevated the percentage of tumor-infiltrating DCs. Despite the lack of alterations in MHC II expression on DCs, the expression of CD80 on these cells was significantly upregulated after MTX or HES-MTX application (Fig. 3I-K). Although, after treatment with MTX or HES-MTX no differences in the percentage of $\mathrm{CD}^{+}{ }^{+} \mathrm{T}$ cells compared to the non-treated group (MC38 nt) were found, a strong, but not statistically significant, 
A

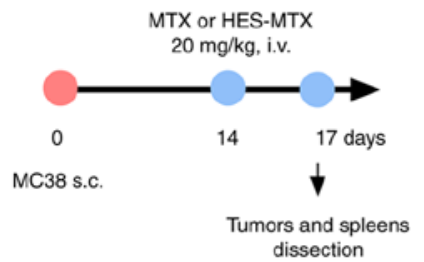

B

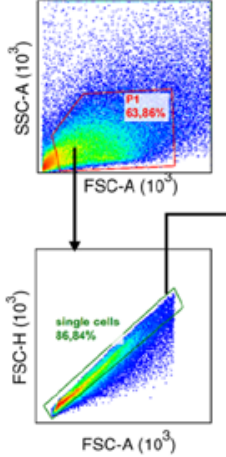
dissection

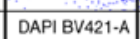

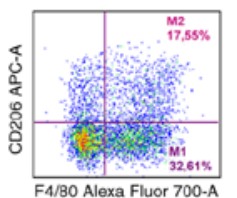
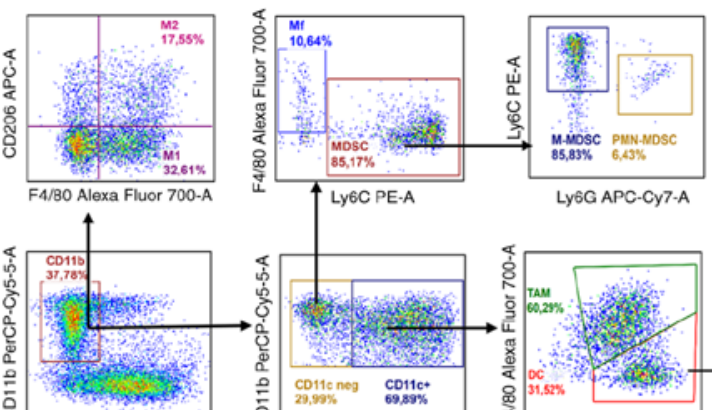

CD3CD19CD49bPE-TexasRed-A
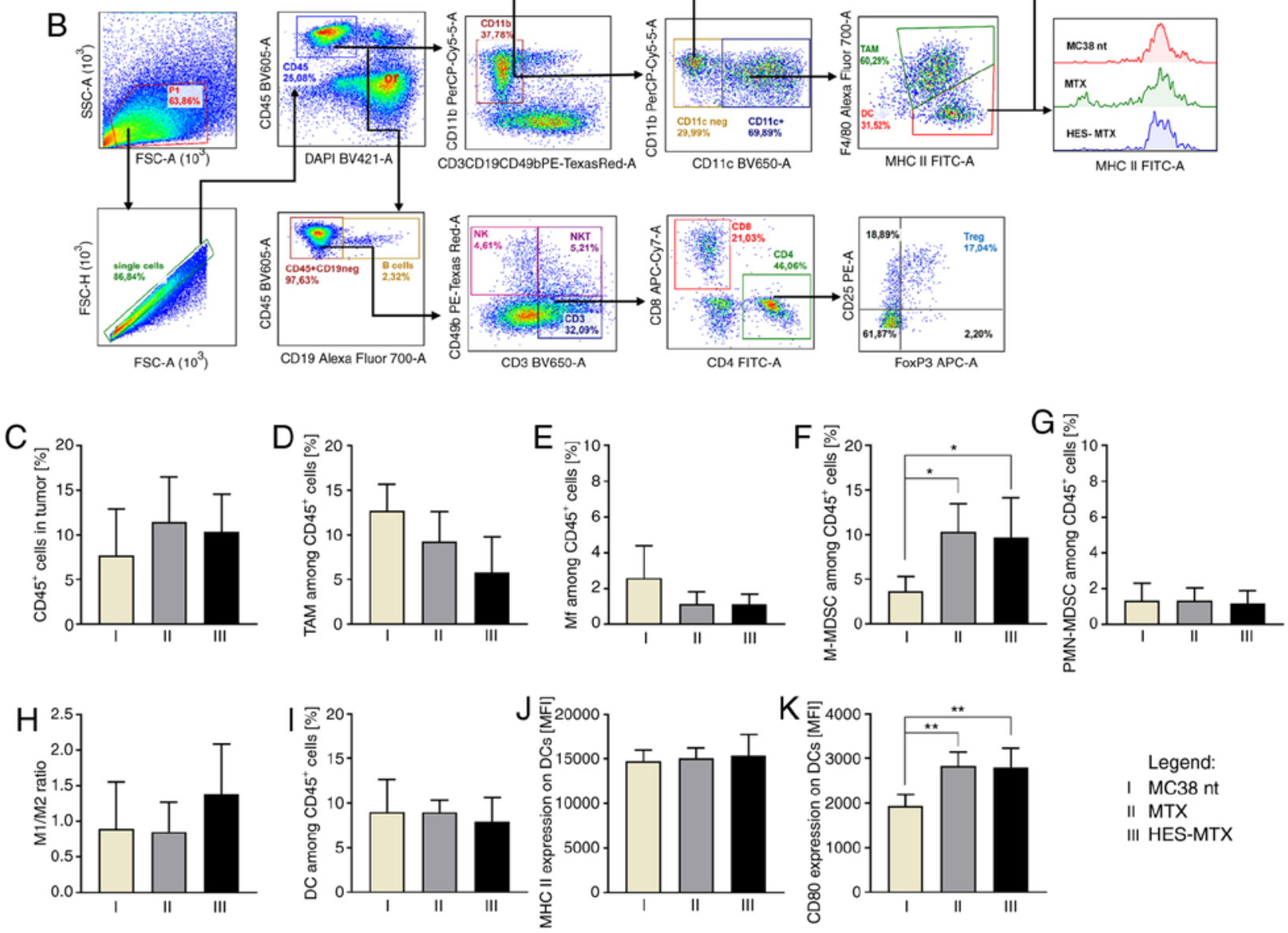

II MTX

III HES-MTX
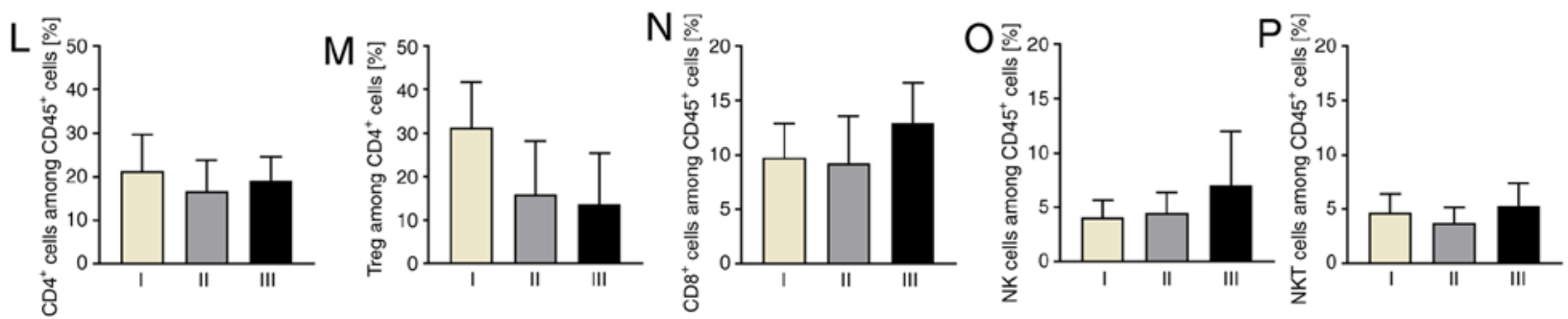

Figure 3. Impact of chemotherapy on infiltration of MC38 tumor nodules with immune cells. (A) Scheme of treatment. (B) Schemes of multiparameter flow cytometry analyses showing the method of distinguishing myeloid or lymphoid cell subpopulation in tumors dissected from MC38 tumor-bearing mice treated according to the scheme presented in A. (C) Percentage of $\mathrm{CD}^{+} 5^{+}$cells in tumor nodules. (D-G) Percentage of myeloid cell subpopulations among CD45 cells in tumors. (H) M1/M2 ratio showing changes in polarization of tumor-infiltrating macrophages after therapy. (I-K) Percentage of DCs infiltrating into tumor tissue and expression of MHC II and CD80 molecules on the surface of DCs. (L-P) Percentage of lymphoid cell subpopulations among CD45 cells in tumors. Results are expressed as mean \pm SD ( 5 mice per group were analyzed from one experiment). In all presented data the differences between groups were calculated using the one-way ANOVA followed by Tukey's multiple comparison post-hoc test $\left({ }^{*} \mathrm{P}<0.05,{ }^{* *} \mathrm{P}<0.01\right)$. HES, hydroxyethyl starch; MTX, methotrexate; DCs, dendritic cells; TAMs, tumor-associated macrophages; Mf, macrophages; M-MDSC, monocytic myeloid derived suppressor cells; PMN-MDSC, polymorphonuclear myeloid derived suppressor cells; Tregs, regulatory T cells; NK, natural killer; i.v., intravenously s.c., subcutaneously.

decrease in the percentage of Tregs in the MTX and HES-MTX groups (Fig. 3L and M) was observed. Moreover, administration of HES-MTX contributed to the increase in the percentage of CD8 ${ }^{+}$T cells, NK and NKT cells (Fig. 3N-P); however, these changes were not statistically significant. The activation status of $\mathrm{CD}^{+}$and $\mathrm{CD}^{+} \mathrm{T}$ cells based on the expression of CD44 and
CD62L antigens was evaluated additionally. The percentage of T cells with effector phenotype $\left(\mathrm{CD} 44^{+} \mathrm{CD} 62 \mathrm{~L}^{\mathrm{neg}}\right)$ and memory phenotype $\left(\mathrm{CD} 44^{+} \mathrm{CD} 62 \mathrm{~L}^{+}\right)$was determined (Fig. S1). The vast majority of $\mathrm{CD}^{+}$(approximately $90 \%$ ) and $\mathrm{CD}^{+}$(approximately $80 \%$ ) $\mathrm{T}$ cells infiltrating into tumor tissue showed effector phenotype $\left(\mathrm{CD} 44^{+} \mathrm{CD} 62 \mathrm{~L}^{\text {neg }}\right)$, however compared to 
A

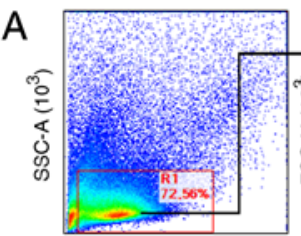

FSC-A $\left(10^{3}\right)$

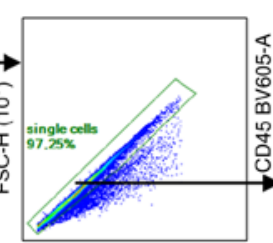

FSC-A $\left(10^{3}\right)$

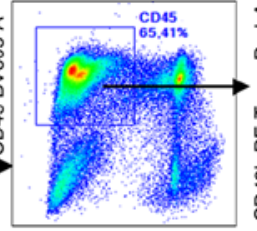

DAPI BV421-A

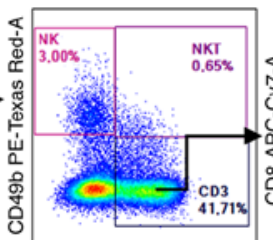

CD3 BV650-A

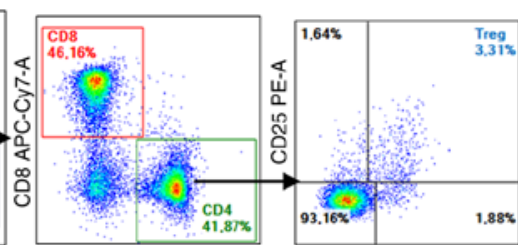

CD4 FITC-A

FoxP3 APC-A
$\mathrm{H}$
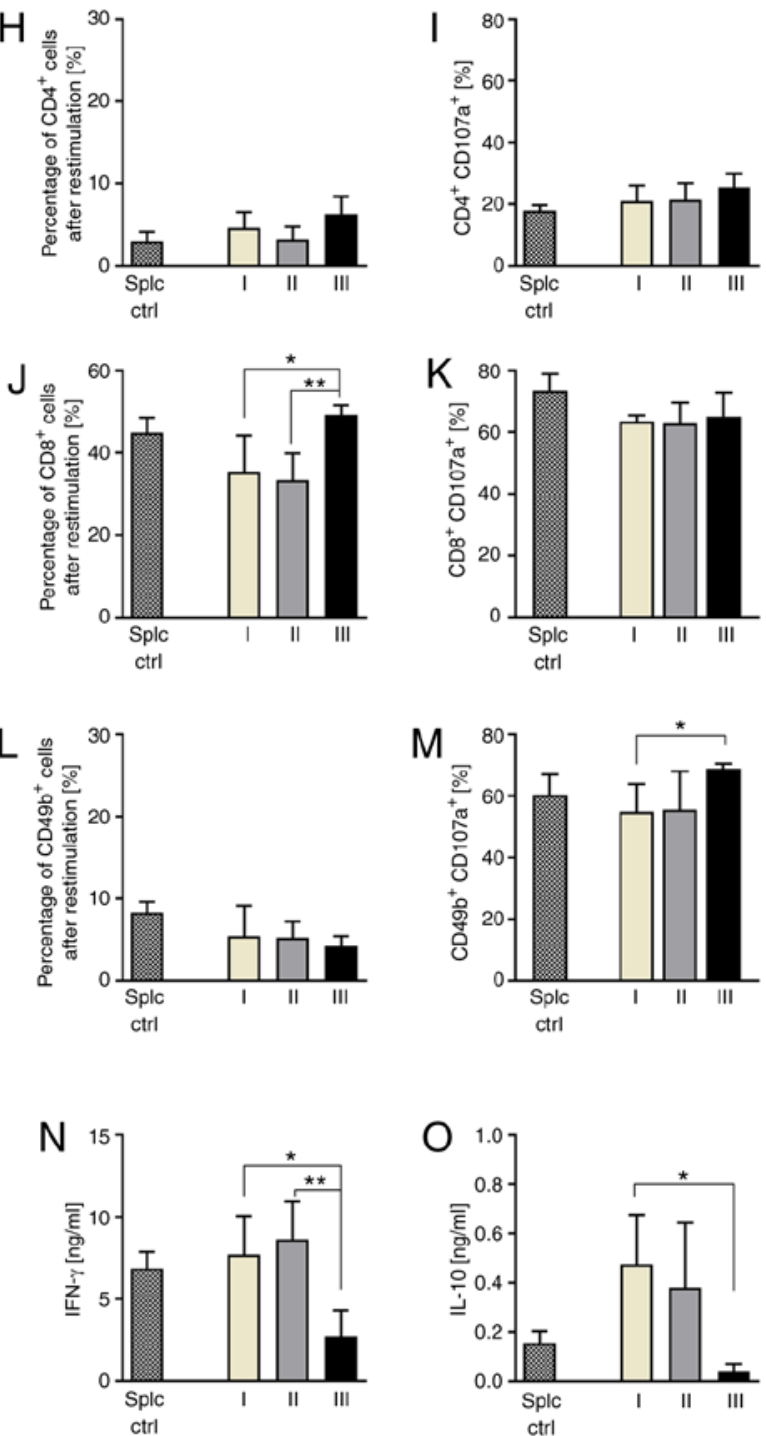
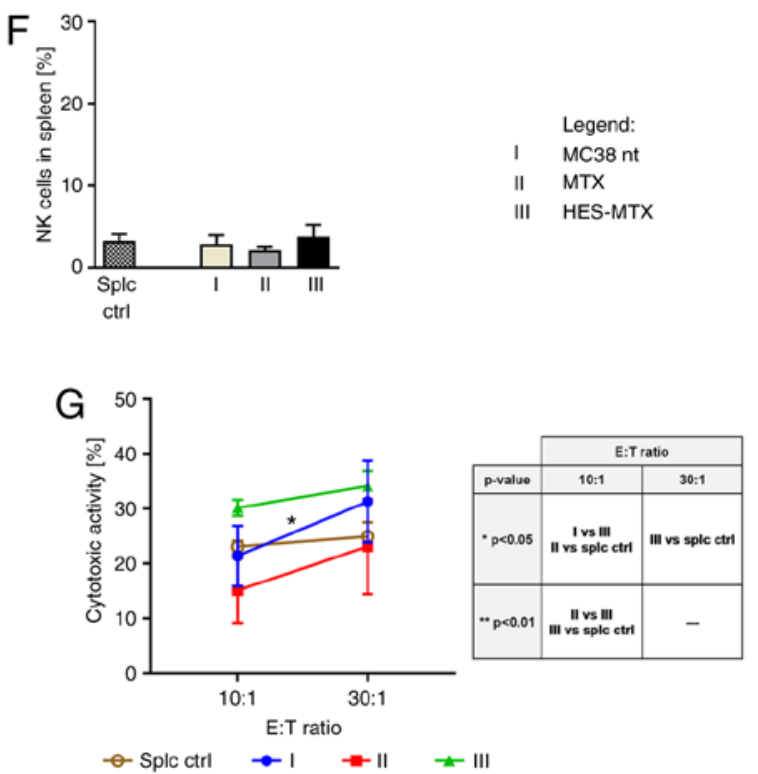

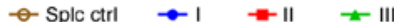

Figure 4. Effect of applied chemotherapy on induction of systemic antitumor response. (A) Scheme of multiparameter flow cytometry analyses showing the method of distinguishing lymphoid cell subpopulation in spleens dissected from MC38 tumor-bearing mice treated according to the scheme presented in Fig. 3A. (B-F) Percentage of effector and suppressor lymphoid cell subpopulations in the spleens. (G) Cytotoxic activity of splenocytes (effector cells) against $\mathrm{DiO}^{+} \mathrm{MC} 38$ cells (target cells). Asterisk above the line indicates statistical significance between different E:T ratios within a given group, while statistical significance between groups within a given E:T ratio is presented in the table. (H-M) Percentage of Th, CTL and NK cells (CD49b $\left.{ }^{+}\right)$among splenocytes after restimulation of spleen cells with MC38 cells and the percentage of CD107a ${ }^{+}$among CD4 ${ }^{+}, \mathrm{CD}^{+}$and CD $49 \mathrm{~b}^{+}$cells measured by CD107a degranulation assay. ( $\mathrm{N}$ and $\mathrm{O}$ ) IFN- $\gamma$ and IL-10 concentration in supernatants after restimulation. Results are expressed as mean \pm SD (5 mice per group were analyzed from one experiment). Splc ctrl, splenocytes isolated from spleen derived from healthy mice (i.e. without MC38-tumor). Differences between groups were calculated using: (B-F and H-M) one-way ANOVA followed by Tukey's multiple comparison post-hoc test (N) nonparametric Kruskal-Wallis test followed by Dunn's multiple comparison test; (O) Brown-Forsythe and Welch ANOVA test followed by Dunnett's T3 multiple comparisons post-hoc test; or (G) two-way ANOVA followed by Tukey's multiple comparison post-hoc test $\left({ }^{*} \mathrm{P}<0.05,{ }^{* *} \mathrm{P}<0.01,{ }^{* * * *} \mathrm{P}<0.0001\right)$. HES, hydroxyethyl starch; MTX, methotrexate; NK, natural killer; Tregs, regulatory T cells; IFN, interferon; IL, interleukin.

the MC38 nt group, the applied therapy did not cause statistically significant changes in the percentage of effector or memory T cells (Fig. S1A-C). The obtained data suggest that although MTX and HES-MTX can reduce the percentage of myeloid (TAM, Mf, PMN-MDSC, M2-type macrophages) and lymphoid (Treg) cells with suppressor activity, only after 
application of HES-MTX was an increase in the percentage of CTL and NK cells noted.

With the use of the multiparametric flow cytometry analysis protocol presented in Fig. 4A, the percentages of CTL, Th and Treg cells among spleen cells were estimated. In contrast to the effects caused by MTX application, the use of HES-MTX induced significant changes in the percentage of lymphocytes among spleen cells (Fig. 4B-F). The application of HES-MTX contributed to a significantly higher percentage of $\mathrm{CD}^{+}$and $\mathrm{CD}^{+} \mathrm{T}$ cells compared to the non-treated group and, in the latter subpopulation, to the MTX-treated group. It should be noted that after HES-MTX application a statistically significant reduction in the Treg percentage among spleen $\mathrm{CD}^{+} \mathrm{T}$ cells was observed. Also, among splenocytes from the HES-MTX-treated group the highest NK and NKT cell percentage was observed. The use of nanoconjugate resulted in the restoration of the size of these lymphocyte populations to the level typical for healthy mice (splc control) or even higher. Analysis of the activation status of splenic $\mathrm{CD}^{+}$and $\mathrm{CD}^{+}$ $\mathrm{T}$ cells revealed that after therapy with the nanoconjugate the percentage of memory $\mathrm{CD} 4^{+} \mathrm{T}$ cells was significantly higher, than after therapy with MTX (Fig. S1D-F). In order to estimate the ability of activated splenic lymphocytes to induce a systemic antitumor response after treatment with HES-MTX, the spleen cells were restimulated ex vivo with MC38 cells. After five days of co-culture of spleen cells with mitomycin C-treated MC38 cells, their cytotoxic activity towards MC38 cells as well as their phenotype, CD107a degranulation and cytokine production were evaluated. Determination of cytotoxic activity of splenocytes against MC38 cells through direct contact revealed statistically significantly higher cytotoxicity in the HES-MTX-treated group compared to the other groups, especially in the 10:1 E:T ratio (Fig. 4G). Similar to observations made during splenocyte phenotype analysis, the elevated percentages of $\mathrm{CD}^{+}{ }^{+}$and $\mathrm{CD}^{+}$cells in the HES-MTX group were maintained after restimulation, and in the latter cell population this difference was statistically significant. It was accompanied by a slight, but not statistically significant, decrease in the percentage of CD49b ${ }^{+}$cells (Fig. 4H, J and L). However, the evaluation of immune cell antitumor activity by CD107a degranulation assay did not show a statistically significant increase in the percentage of $\mathrm{CD}^{+} \mathrm{CD} 107 \mathrm{a}^{+}$cells in the HES-MTX group, while the percentage of $\mathrm{CD} 8^{+} \mathrm{CD} 107 \mathrm{a}^{+}$cells remained unchanged in all groups. It should be highlighted that therapy with the nanoconjugate caused a significant increase in the percentage of $\mathrm{CD} 49 \mathrm{~b}^{+} \mathrm{CD} 107 \mathrm{a}^{+}$cells (Fig. 4I, K and M). Moreover, in the HES-MTX group a significant decrease in IFN- $\gamma$ and IL-10 production after restimulation was observed (Fig. 4N and $\mathrm{O}$ ).

The results presented above suggest that three days after chemotherapy application, especially when HES-MTX was used, the modulation of antitumor response occurred. It was reflected in a reduction in the size of the population of immune cell with suppressor activity (Tregs, TAMs, Mfs and M2-type macrophages) in tumors, as well as in an expansion in the population of immune cells with cytotoxic activity $\left(\mathrm{CD}^{+}\right.$ T cells, NK, NKT cells) in spleen and tumor tissues. Moreover, generation of cytotoxic activity of spleen lymphocytes against tumor cells indicated that HES-MTX contributed to activation of the systemic antitumor immune response.
Antitumor activity of therapy composed of HES-MTX and DC-based vaccines and its influence on local and systemic antitumor immune response. Taking into consideration the immunomodulatory activity of HES-MTX, in the next step of the research, the nanoconjugate was applied in combination with DC-based vaccines according to the scheme presented in Fig. 5A. The tumors and spleens were dissected at two time points: From non-treated (MC38 nt), MTX and HES-MTX groups on the 31st day, and from immunotherapy receiving groups (DC/TAg, MTX+DC/TAg, HES-MTX+DC/TAg) on the 35 th day of the experiment. The time discrepancies were caused by different tumor growth rates, especially in the non-treated group and MTX group. Thus, in the course of the experiment, we decided to separate the dissections-the major reason for this decision was prolonged observation of tumor growth after the last injection of DC-based vaccines and maintaining the interval between organ dissections as short as possible. The tumor growth inhibition (TGI) calculated on the 30 th day of the experiment for all groups of mice indicated the strongest influence of HES-MTX on tumor growth (the TGI in HES-MTX group was $64 \%$ in relation to the MC38 nt group), while MTX exhibited only 6\% inhibition of tumor growth (Fig. 5B). A moderate effect was observed after application of the DC-based vaccines as sole therapy (DC/TAg; TGI 39\%). However, when compared to the HES-MTX, group combining the chemotherapy with DC/TAg-based vaccines did not contribute to the enhancement of tumor growth inhibition as we expected. In the HES-MTX+DC/TAg group the TGI was $55 \%$, whereas in the MTX+DC/TAg group the inhibition of tumor growth was negligible (TGI 12\%). The kinetics of MC38 tumor growth and median tumor volume on the 30th day of the experiment are shown in Fig. 5C and D.

To compare the effect of multiple injections of DC-based vaccines, the TGI for the 35th day of the experiment was determined in relation to the DC/TAg group (Fig. S2A). TGI for the MTX+DC/TAg group was negative and was $-14 \%$; hence application of MTX prior to DC-based vaccines probably reduced the effectiveness of the immunotherapy. On the other hand, in the HES-MTX+DC/TAg group, TGI for the 35th day of the experiment was $22 \%$, which indicates that HES-MTX application prior to the start of immunotherapy enhanced tumor growth delay compared to the DC/TAg group, although these differences were not statistically significant. This was also reflected in the median tumor volume on the 35 th day (Fig. S2B).

The multiparameter flow cytometry analyses of tumor tissue (according to the scheme presented in Fig. S3A) showed the overall increase in the percentage of leukocytes in all treated groups of mice (Fig. 5E). Although after monotherapy (MTX, HES-MTX, DC/TAg groups) the percentage of $\mathrm{CD} 45^{+}$cells was slightly higher than in the non-treated group, application of combined therapy, both MTX+DC/TAg and HES-MTX+DC/TAg, caused the most intensive influx of the cells and observed changes were statistically significant in comparison to the MC38 nt group. One of the most numerous cell populations among myeloid cells found in the tumor tissue was tumor-associated macrophages (TAMs), which accounted for approximately $30 \%$ of all leukocytes in tumors from the non-treated group of mice (Fig. 5F). The applied therapy, both chemo- and chemoimmunotherapy, did 

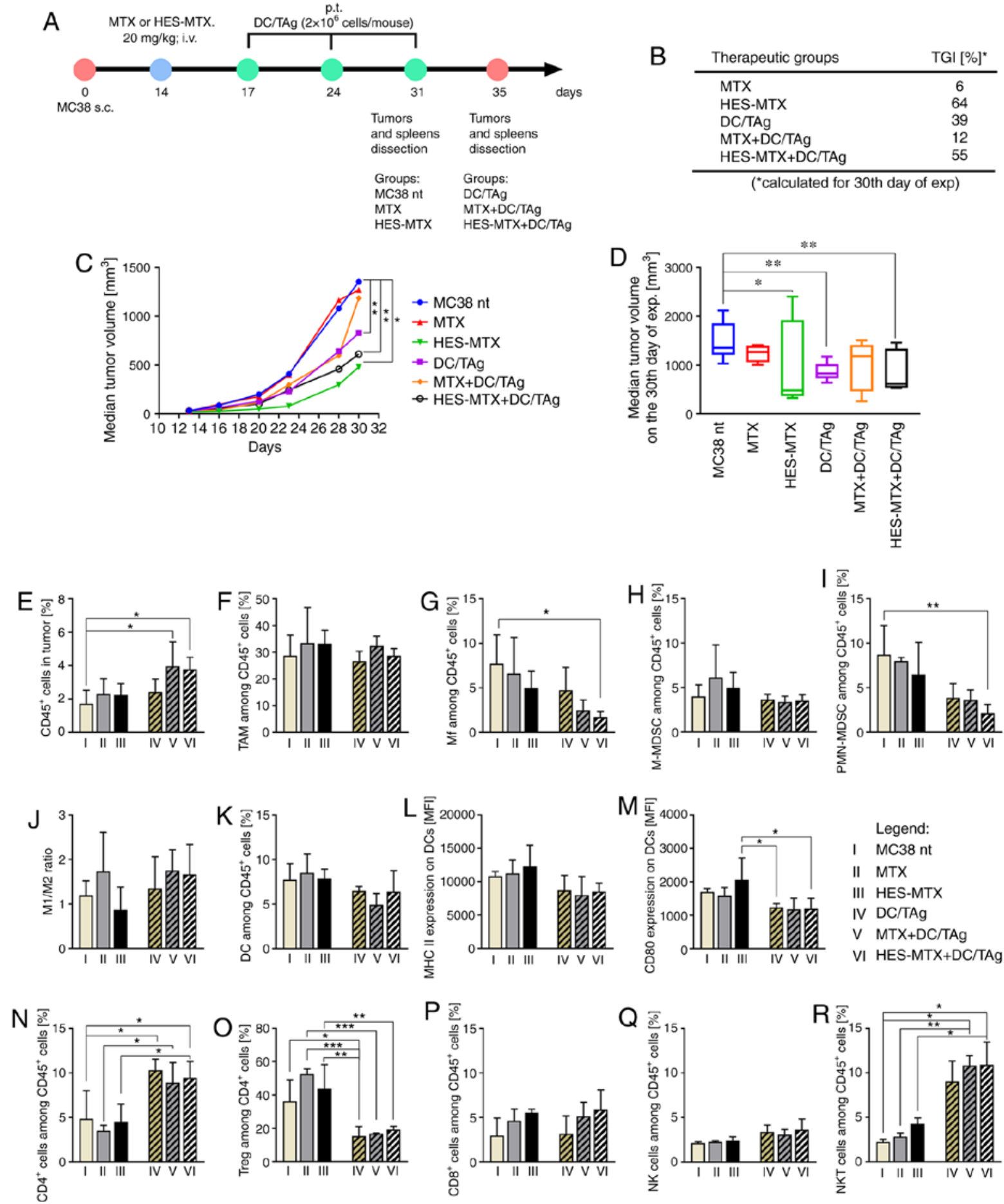

Figure 5. Impact of combined therapy on tumor growth and infiltration of MC38 tumor nodules with immune cells. (A) Scheme of treatment. (B) Table presenting MC38 tumor growth inhibition (TGI) calculated on 30th day of experiment in relation to the MC38 nt group; (C) Graph presenting median tumor volume after chemoimmunotherapy. (D) Box graph presenting median tumor volume, calculated on the 30th day of the experiment. (E) Percentage of CD45 cells in tumor nodules. (F-I) Percentage of myeloid cell subpopulations among CD45 cells in tumors. (J) M1/M2 ratio showing changes in polarization of tumor-infiltrating macrophages after therapy. (K-M) Percentage of DCs infiltrating into tumor tissue and expression of MHC II and CD80 molecules on their surface. (N-R) Percentage of lymphoid cell subpopulations among $\mathrm{CD} 45^{+}$cells in tumors. Scheme of multiparameter flow cytometry analyses showing the method of distinguishing myeloid or lymphoid cell subpopulation in tumors is presented in Fig. S3A. Results are expressed as mean \pm SD (3-5 mice per group were analyzed from one experiment). The differences between groups were calculated by (C and D) two-way ANOVA followed by Bonferroni's multiple comparisons test, (E-Q) one-way ANOVA followed by Tukey's multiple comparison post-hoc test or (R) Brown-Forsythe and Welch ANOVA test followed by Dunnett's T3 multiple comparisons post-hoc test $\left({ }^{*} \mathrm{P}<0.05,{ }^{* *} \mathrm{P}<0.01,{ }^{* * *} \mathrm{P}<0.001\right)$. HES, hydroxyethyl starch; MTX, methotrexate; DCs, dendritic cells; TAg, tumor antigen; TAMs, tumor-associated macrophages; Mf, macrophages; M-MDSC, monocytic myeloid derived suppressor cells; PMN-MDSC, polymorphonuclear myeloid derived suppressor cells; Tregs, regulatory T cells; NK, natural killer; i.v., intravenously s.c., subcutaneously.

not induce changes in the TAM influx. However, the applied therapies demonstrated a significant impact on decrease in the percentage of resident Mfs in the tumor nodules, especially in the HES-MTX+DC/TAg group, in which the Mf percentage 
was about four times lower than that noted in the MC38 non-treated group and three times lower than in the DC/TAg group (Fig. 5G). Although there were no statistically significant changes in size of the M-MDSC population-an increase in the M-MDSC percentage occurred only when MTX or HES-MTX was applied as monotherapy; significant changes in the PMN-MDSC percentage in tumor tissue were observed (Fig. 5H and I). After application of each type of therapy, a reduced population of PMN-MDSCs was found. Although application of chemotherapy alone caused a moderate decrease of PMN-MDSC percentage (especially after HES-MTX), the use of immunotherapy induced a significant reduction in the PMN-MDSC percentage. The lowest percentage of these cells was noted after combined therapy with HES-MTX and DC-based vaccines. Considering the influence of applied therapies on the stage of macrophage polarization, the M1/M2 ratio was not significantly increased after application of MTX or combined therapy (MTX+DC/TAg, HES-MTX+DC/TAg group), while it decreased after HES-MTX treatment (Fig. 5J). In comparison to non-treated and chemotherapy groups, DC-based vaccines caused a slight, but not statistically significant reduction in the percentage of DCs infiltrating tumor tissue, which was accompanied by decreased expression of MHC II and CD80 molecules on their surface (Fig. 5K-M). Despite the fact that the lowest percentage of DCs was found in the MTX+DC/TAg group, the expression of MHC II and CD80 antigens did not change and remained at the same level as in the DC/TAg-receiving group. It should be noted that in the HES-MTX group, tumor-infiltrating DCs were characterized by the highest expression of MHC II and CD80 molecules, which is consistent with our observations about the modulatory potential of the nanoconjugate for the DC phenotype, and observed changes in the expression of CD80 antigen were statistically significant.

The changes occurring in the myeloid populations were accompanied by modifications in the percentage of lymphoid cell infiltrating tumor nodules. When compared to the MC38 nt group, the use of cytostatics alone did not cause statistically significant changes in the percentage of $\mathrm{CD}^{+}$ cells infiltrating tumor tissue, unlike in other lymphoid cell subpopulations. Application of DC-based vaccines resulted in statistically significant enlargement of $\mathrm{CD}^{+} \mathrm{T}$ cells, NKT cell percentage and a decrease in the percentage of Tregs, while the increase of NK cells percentage was not statistically significant (Fig. 5N, O, Q and R). Trends in changes in the percentage of Tregs suggest that application of chemotherapeutics alone was not sufficient to maintain the size of the tumor-infiltrating Treg population at a low level, like those observed on the third day after chemotherapy in the previous experiment. In comparison to the control group, the percentage of Tregs cells was much higher, especially in the MTX group (Fig. 5O). Multiple application of DC-based vaccines was found to cause a statistically significant strong reduction in the Treg percentage, but in the HES-MTX+DC/TAg group the size of the Treg population was slightly greater than that noted in the other DC/TAg-receiving groups. It is noteworthy that an increase in $\mathrm{CD}^{+} \mathrm{T}$ cells was noted when chemotherapy was applied alone (MTX, HES-MTX groups) or in combination with DC-based vaccines (MTX+DC/TAg, HES-MTX+DC/TAg) (Fig. 5P), but these changes were not statistically significant. The highest percentage of $\mathrm{CD}^{+} \mathrm{T}$ cells was noted in the HES-MTX-receiving groups (HES-MTX and HES-MTX+DC/TAg), which is consistent with our previous observation that HES-MTX affects the enhanced influx of $\mathrm{CD}^{+} \mathrm{T}$ cells into tumor tissue. Furthermore, in comparison to the control or DC/TAg group, a significant increase in the percentage of NKT cells was observed when MTX or HES-MTX was used (as mono- and combined therapy) (Fig. 5R). Analysis of the activation status of $\mathrm{CD}^{+} \mathrm{T}$ cells infiltrating into tumor tissue showed that compared to the non-treated and chemotherapy-receiving groups (MC38 control, MTX and HES-MTX), the use of DC/TAg-based vaccines resulted in a significantly higher percentage of effector $\mathrm{CD}^{+} \mathrm{T}$ cells, while percentage of memory $\mathrm{CD} 4^{+}$ $\mathrm{T}$ cells was decreased. Moreover, therapy consisting of HES-MTX and DC/TAg caused a significant increase in the percentage of effector $\mathrm{CD} 8^{+} \mathrm{T}$ cells infiltrating into the tumor tissue (Fig. S4B and C).

The obtained results indicated that supplementing the chemotherapy with DC-based vaccines contributed to an enhanced influx of leukocytes into the tumor tissue, especially CTL and NKT cells, and reduced the population of immune cells with suppressor activity, such as Mfs, PMN-MDSCs and Tregs.

The estimation of the percentage of CTLs, Th and Tregs among spleen cells (according to the scheme presented in Fig. S3B) revealed that significant changes in the lymphoid cell population occurred only when DC-based vaccines were used (Fig. 6A-E). In comparison to the non-treated group, the application of MTX or HES-MTX as monotherapy did not contribute to significant alterations among CTLs, Th or Tregs, like those observed on the third day after chemotherapy. When DC-based vaccines were applied alone, we did not observe significant changes in the population size of the mentioned lymphocytes in the spleens. However, therapy consisting of HES-MTX and DC/TAg was found to cause a decrease in the percentage of $\mathrm{CD}^{+}$and $\mathrm{CD}^{+} \mathrm{T}$ cells compared to the non-treated and DC/TAg groups. This effect was accompanied by a significant increase in the Treg percentage in this group. Similar tendencies were observed in the MTX+DC/TAg group. Among the DC/TAg-receiving groups, the highest percentage of splenic effector $\mathrm{CD}^{+} \mathrm{T}$ cells was observed in the HES-MTX+DC/TAg group and this change was statistically significant compared to the DC/TAg and MTX+DC/TAg group. Moreover, in the case of memory $\mathrm{CD} 8^{+} \mathrm{T}$ cells found in the spleen, after use of the DC/TAg-based vaccines the percentage of these was significantly lower than observed in the non-treated and HES-MTX groups (Fig. S4E and F).

It should be noted that assessment of cytotoxic activity of restimulated spleen cells towards MC38 tumor cells revealed that immunotherapy generated more efficient cytotoxic activity than chemotherapy applied alone (Fig. 6F). Moreover, the highest cytotoxic activity was noted in the HES-MTX+DC/TAg group in both E:T ratios. The restimulation of splenocytes by MC38 cells confirmed the impact of DC-based vaccines on the percentage of $\mathrm{CD}^{+}, \mathrm{CD}^{+}$and $\mathrm{CD} 49 \mathrm{~b}^{+}$cells, while chemotherapy applied alone did not cause any significant changes in these subpopulations (Fig. 6G, I and K). Despite the lack of alterations in the $\mathrm{CD}^{+} \mathrm{T}$ cell percentage after restimulation between groups receiving DC-based vaccines, a strong 

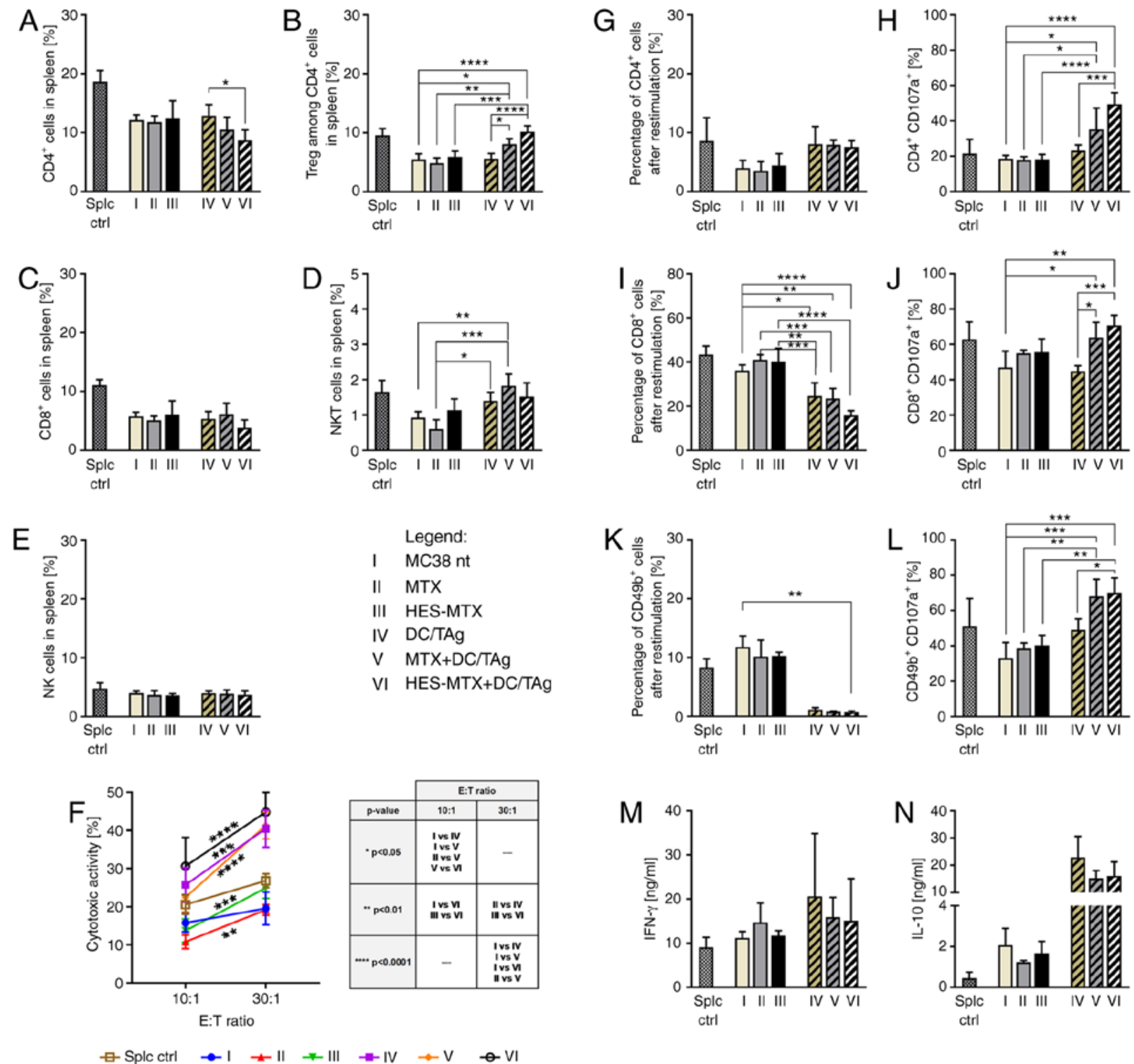

Figure 6. Effect of applied chemoimmunotherapy on induction of systemic antitumor response. (A-E) Percentage of effector and suppressor lymphoid cell subpopulations in spleens of MC38 tumor-bearing mice treated according to the scheme presented in Fig. 5A. (F) Cytotoxic activity of splenocytes (effector cells) against $\mathrm{DiO}^{+} \mathrm{MC} 38$ cells (target cells). Asterisks above or under the lines indicate statistical significance between different E:T ratios within a given group, while statistical significance between groups within a given E:T ratio is presented in the table. (G-L) Percentage of Th, CTL and B NK cells (CD49b ${ }^{+}$ among splenocytes after restimulation of spleen cells with MC38 cells and the percentage of CD107a ${ }^{+}$among CD4 $4^{+}$, and cytotoxic CD8 ${ }^{+}$and CD49b cells measured by CD107a degranulation assay. (M and N) IFN- $\gamma$ and IL-10 concentration in supernatants after restimulation. Scheme of multiparameter flow cytometry analyses showing the method of distinguishing lymphoid cell subpopulation in spleens is presented in Fig. S3B. Results are expressed as mean \pm SD (3-5 mice per group were analyzed from one experiment). Splc ctrl, splenocytes isolated from spleen derived from healthy mice (i.e. without MC38-tumor). Differences between groups were calculated using: (A-E, G-J and L) one-way ANOVA followed by Tukey's multiple comparison post-hoc test (K, M and N) the nonparametric Kruskal-Wallis test followed by Dunn's multiple comparison test; or (F) two-way ANOVA followed by Tukey's multiple comparison post-hoc test $\left({ }^{*} \mathrm{P}<0.05,{ }^{* * *} \mathrm{P}<0.01,{ }^{* * * *} \mathrm{P}<0.001,{ }^{* * * * *} \mathrm{P}<0.0001\right)$. HES, hydroxyethyl starch; MTX, methotrexate; DCs, dendritic cells; TAg, tumor antigen; Tregs, regulatory T cells; Th, T helper; CTL, cytotoxic T lymphocyte; NK, natural killer; IFN, interferon; IL, interleukin.

reduction in the population size of $\mathrm{CD}^{+} \mathrm{T}$ cells and $\mathrm{CD} 49 \mathrm{~b}^{+}$ cells was found, especially in the HES-MTX+DC/TAg group. Nevertheless, the CD107a degranulation assay demonstrated that application of combined therapy caused a robust increase in the percentage of $\mathrm{CD} 107 \mathrm{a}^{+}$cells among the $\mathrm{CD} 4^{+}$, $\mathrm{CD}^{+}$and $\mathrm{CD} 49 \mathrm{~b}^{+}$cells (Fig. $6 \mathrm{H}, \mathrm{J}$ and $\mathrm{L}$ ). Application of HES-MTX together with multiple injections of DCs induced the highest percentage of $\mathrm{CD} 4{ }^{+} \mathrm{CD} 107 \mathrm{a}^{+}, \mathrm{CD} 8^{+} \mathrm{CD} 107 \mathrm{a}^{+}$and $\mathrm{CD} 49 \mathrm{~b}^{+} \mathrm{CD} 107 \mathrm{a}^{+}$cells and these changes were statistically significant. However, in the latter subpopulation, a similar effect was observed in the MTX+DC/TAg group. This effect was accompanied by increased production of IFN- $\gamma$ and IL-10 in all immunotherapy-receiving groups (Fig. 6M and N).

The gathered data indicate that although monotherapy with the DC-based vaccine also generated alterations in certain crucial immune cell subpopulations in tumor nodules and spleens, the augmentation of these effects was observed when chemotherapy was applied prior to immunotherapy. Combined therapy with HES-MTX and DC-based vaccines resulted in a reduction in percentages of myeloid cells with suppressor activity in infiltrating tumor and enhanced influx of CD8, NK and NKT cells. Moreover, therapy consisting of HES-MTX 
and DC-based vaccines contributed to generation of a specific antitumor response. It was confirmed by increased ability of spleen cells to secrete cytolytic granules and enhanced cytotoxic activity as a result of secondary contact with the tumor antigen. All of these factors caused the statistically significant delay of tumor growth after therapy with HES-MTX and DC-based vaccines.

\section{Discussion}

In the present work, we demonstrated for the first time that an innovative drug delivery system-in the form of a nanoconjugate of well-known therapeutic compounds, i.e. methotrexate (MTX) as an anticancer agent and hydroxyethyl starch (HES) as a high-molecular carrier-was able to modulate the antitumor immune response. Moreover, we were the first to apply chemotherapy with the HES-MTX nanoconjugate together with DC-based cellular vaccines in a murine MC38 colon carcinoma model.

According to Goszczyński et al the mean hydrodynamic diameter of HES-MTX is $15.2 \pm 6.2 \mathrm{~nm}$ (20); therefore undoubtedly this type of conjugate can be defined as a nanoconjugate. It is noteworthy that conjugation of MTX with HES is achieved by esterification of HES's hydroxyl groups, but the linker between the carrier and MTX is glutamic acid-an integral part of the MTX molecule- and hence no other additional linking substances are needed (20). MTX release from the nanoconjugate occurs as a result of chemical and enzymatic hydrolysis by esterases or amylases. Enzymatic degradation of HES leads to the release of glucose derivatives only, allowing for easy elimination of these derivatives from the body. At the beginning of the research on the anticancer potential of HES-MTX, the key issue was to determine what the main advantage of HES-MTX over the free form of MTX is. In fact, chemotherapy in its conventional form, including methotrexate application, is related to overall toxicity to healthy cells, rapid elimination of chemotherapeutics from the body and low specificity towards target cancer cells $(23,48)$. Thus, it was challenging to design a chemotherapeutic-carrier system overcoming these difficulties. It is well known that MTX enters the cell mainly through the ubiquitously expressed reduced folate carrier (RFC) to which MTX has high affinity. MTX can also enter cells via folate receptors (FRs) overexpressed on cancer cells, although with low affinity (27). As a result of conjugation of one molecule of hydroxyethyl starch with 50 molecules of MTX, the nanoconjugate becomes polyvalent $(17,28)$. This polyvalence allows interactions of the nanoconjugate with folate receptor alpha $(\mathrm{FR} \alpha)$ with a much higher binding constant than free MTX, and therefore we postulate that HES-MTX interacts more strongly with the tumor cells overexpressing FR $\alpha$ than normal cells. Another important advantage of HES-MTX is its biodistribution, which is attained not only, although mainly, by interaction of HES-MTX with FRs on target cells but also by an enhanced vascular permeability and retention effect (EPR). This phenomenon is considered as an ability of macromolecules larger than $40 \mathrm{kDa}$ (hydrodynamic diameter above $10 \mathrm{~nm}$ ) to selectively leak from tumor vessels and accumulate in tumor tissue (2,30-32). EPR is often observed in solid tumors due to extensive angiogenesis, malfunctional vascular architecture and increased expression of proteins associated with vascular permeability $(49,50)$. Moreover, the use of a carrier reduces the toxicity of therapy, since the EPR effect for drug delivery does not occur in normal tissue.

The main objective of the present study was to determine whether the HES-MTX nanoconjugate, applied as chemotherapy, modulates the systemic antitumor immune response, and affects changes in the landscape of immune cells infiltrating tumor tissue. This, in turn, should support the generation of a proper immune response against a growing tumor by DC-based vaccines injected peritumorally after chemotherapy administration. Furthermore, there are reports confirming that certain cytostatics, including methotrexate, used at appropriate doses, can act as modulators of the DC phenotype and function $(33,34)$, thus DCs reinforced in this way should generate an efficient antitumor immune response (51).

Taking all the above into account, we designed in vitro studies in which we found that the antiproliferative activity of the HES-MTX nanoconjugate against MC38 colon carcinoma cells was considerably lower than that of the free form of MTX. Previous results reported by Goszczyński et al revealed that HES-MTX possessed approximately 10-fold weaker antiproliferative activity towards human (MV4-11) and murine (P388) leukemia cell lines than MTX alone (20), which is consistent with our observations. Nevertheless, weak in vitro efficiency of the conjugates does not necessarily predict diminished in vivo activity, as it has been shown for the fibrinogen-MTX conjugate or dextran-MTX conjugate used in a P388 mouse leukemia model by Nevozhay et al (44) or Goszczyński et al (14), respectively. Furthermore, we confirmed the interaction of HES-MTX labelled with FITC dye with MC38 cells and dendritic cells (DCs). In comparison to MC38 cells, DCs interacted more strongly with HES and HES-MTX. It was reflected in greater MFI values for DCs, but it may be associated with increased antigen uptake capacity, which is typical for this type of cell. We also confirmed a high affinity of nanoconjugate to folate binding protein and we verified the overexpression of FR $\alpha$ in MC38 cells in comparison to DCs ex vivo generated from murine bone marrow precursors.

In the next step in the in vitro research, we estimated the influence of metabolites released by MC38 cells treated with nanoconjugate on the generation and maturation of DCs. It is well known that in the presence of the tumor microenvironment, DC functions are hindered and thereby creation of an efficient antitumor immune response by DCs is impaired $(8,9,52)$. It has also been confirmed by us that in comparison to untreated DCs, murine bone marrow DC precursors cultured in the presence of $\mathrm{CM}$ harvested from above MC38 cells responded more weakly to stimulation with TAg (DC/MC38/TAg), which was demonstrated in statistically significantly lower expression of surface molecules necessary for efficient antigen presentation. Using the Annexin V binding assay, we confirmed that HES-MTX induced weaker apoptosis of MC38 cells than MTX. DC precursors cultured in CM harvested from above MC38 cells treated with HES-MTX and stimulated with TAg (DC/MC38/HES-MTX/TAg) exhibited modest changes in expression of DC antigens in comparison to DC/MC38/MTX/TAg. It was associated with lower toxicity of HES-MTX, than MTX, towards tumor cells. Therefore, in relation to DC/MC38/MTX/TAg, only partial abolition of 
the negative effect of tumor cell metabolites on DC generation and maturation was observed. It should be highlighted that in contrast to MTX, DCs cultured in the presence of HES-MTX and stimulated with TAg (DC/HES-MTX/TAg) were characterized by statistically significant elevated expression of costimulatory molecules, which indicates that tumor antigens would be more efficiently presented to naïve lymphocytes by these cells. Similar results were also described by other researchers, who showed that certain chemotherapeutic agents such as methotrexate, paclitaxel or doxorubicin used in low, noncytotoxic concentrations during DC generation can upregulate maturation, antigen processing, and antigen presentation by DCs, and this phenomenon was called chemomodulation (33). For instance, Shurin et al reported that MTX present during murine DC differentiation not only contributed to an increase in expression of antigen-processing machinery proteins and costimulatory molecules on these cells, but also resulted in upregulated expression of IL-12p70 in DCs. In turn, this cytokine enhanced the ability of murine $\mathrm{DCs}$ to present antigens to $\mathrm{T}$ cells in vitro (33). Moreover, Kaneno and co-workers found that human DCs cultured in the presence of methotrexate showed increased expression of costimulatory molecules. Furthermore, the ability of these DCs to stimulate proliferation of allogeneic $\mathrm{T}$ lymphocytes was also increased (35). In addition, Zhong et al demonstrated that paclitaxel used in appropriate low doses supported murine DC maturation and function, and -importantly-pretreatment of 3LL cells with paclitaxel abrogated the suppressive effect of the tumor milieu on DC generation (53).

Altogether, the main advantage of using the nanoconjugate rather than MTX in free form is the fact that the physicochemical properties of HES-MTX make it possible to target tumor cells and prolong accumulation of the nanoconjugate in tumor tissue. Moreover, the nanoconjugate itself modulates the phenotype of DCs and improves the maturation of these cells, and thus it may contribute to generation of an efficient antitumor immune response by DCs present in the body as well as by DCs administered in the form of cellular vaccines. This knowledge allowed us to put forward a hypothesis that more efficient accumulation of HES-MTX in tumor tissue and greater tumor cell specificity (despite the lower antiproliferative activity towards MC38 cells in vitro) will affect the efficacy of HES-MTX in vivo. It should be reflected not only by enhanced inhibition of the growing tumor in comparison to MTX, but also by modulation of the ability of DCs to elicit an effective antitumor immune response by the host's immune system.

Taking into consideration the above results, our primary purpose in the subsequent experiments was to combine anticancer therapy with the nanoconjugate and multiple peritumoral injection of DC-based vaccines in a murine colon carcinoma model. In our previous chemoimmunotherapy schedules in this tumor model, the DC-based vaccines were applied three days after cyclophosphamide administration $(39,40,46)$. Moreover, certain chemotherapeutics, such as MTX $(1,19)$, paclitaxel $(36)$ or cyclophosphamide $(37,38)$, may act as immunomodulators through stimulation of effector immune cells and elimination of regulatory T cells (Tregs) $(39,40,54)$. Thus, it was necessary to find out whether HES-MTX administration would change the local and systemic antitumor response and how it would affect the activity of DC-based vaccines injected three days after chemotherapy.

In the tumor nodules dissected three days after MTX or HES-MTX application, an increase in leukocyte influx was observed. Nevertheless, only the HES-MTX treatment contributed to polarization of tumor-infiltrating macrophages towards M1-type cells, and greater influx of cytotoxic T lymphocytes (CTLs) and natural killer (NK) cells into tumor tissue occurred. Regardless of that, after MTX and HES-MTX treatment, the percentage of monocytic myeloid derived suppressor cells (M-MDSCs) in tumors was significantly elevated, the size of the other cell populations with potent suppressor activity i.e. macrophages (Mfs), tumor-associated macrophages (TAMs) and Tregs, was reduced and importantly, in the HES-MTX group the percentages of TAMs and Tregs were the lowest; however, all the above-mentioned differences were not statistically significant.

Considering the influence of HES-MTX on the tumor-directed systemic immune response, we observed a significant increase of T helper (Th), CTL and NKT cell percentages among splenic leukocytes, and this effect was accompanied by a decrease in the percentage of Tregs among splenic $\mathrm{CD}^{+} \mathrm{T}$ cells. Despite the substantial decrease in the production of interferon (IFN)- $\gamma$ and interleukin (IL)-10 by restimulated splenocytes obtained from mice treated with HES-MTX, we observed higher cytotoxic activity towards tumor cells in this group. Thus, we postulate that this resulted from the higher percentage of $\mathrm{CD}^{+} \mathrm{T}$ cells and an increase in the percentage of $\mathrm{CD} 49 \mathrm{~b}^{+} \mathrm{CD} 107 \mathrm{a}^{+}$after restimulation. These observations confirm that the use of the methotrexate nanoconjugate not only can affect the systemic immune response by activation of a specific antitumor response, but also can change the landscape of tumor-infiltrating immune cells from an unfavorable environment. This in turn should contribute to creation of more appropriate conditions for generation of a specific antitumor immune response by DCs inoculated peritumorally, the use of which was planned in further experiments.

These findings led us to the next stage of in vivo studies in which we estimated the antitumor activity of combined chemoimmunotherapy, not only by defining the alterations occurring in the antitumor immune response, but also by determining the effect of the therapy by tumor growth inhibition (TGI) calculation. Despite the different tumor growth rate in the MC38 non-treated (control) group and MTX group and, as consequence of this, dissections of organs at two time points, we were able to define TGI for the 30th day of the experiment as a common denominator for all tested groups. As a result of applied monotherapy with HES-MTX the TGI value was $64 \%$, but extending the treatment scheme by multiple peritumoral injection of DC/TAg did not improve the therapeutic effect of HES-MTX (TGI was 55\%). Based on our previous studies on the use of combined therapy composed of cyclophosphamide (CY) and DC-based vaccines in this tumor model (46), we postulate that the lower TGI value observed in the HES-MTX+DC/TAg group (compared to the HES-MTX group) may be related to other suppressive factors, e.g. cytokines, which are present in the tumor microenvironment (TME). We made such an observation in a previous study, Rossowska et al (46) where CY administration 
generated slightly higher TGI than combined treatment with CY+DC/TAg. Thus, we hypothesize that weaker effectiveness of therapy consisting of nanoconjugate and DC/TAg-based vaccines might be associated with TME-derived immunosuppressive factors, e.g. IL-10, which affect the function of DCs administered as cellular vaccines.

The enhancement of the CD45 cell influx into tumor nodules only after combined therapies $(\mathrm{MTX}+\mathrm{DC} / \mathrm{TAg}$ and HES-MTX+DC/TAg groups) again indicates that the application of chemotherapy prior to DC injection generates a favorable immune microenvironment in tumors. This was also reflected in the insignificantly increased M1/M2 ratio value. The lowest percentage of Mfs and polymorphonuclear (PMN)-MDSCs was found in the HES-MTX+DC/TAg group. The decrease in the percentage of DCs infiltrating into tumor tissue in the DC/TAg-receiving group of mice suggests the intensified migration of in situ activated DCs to draining lymph nodes. Moreover, the highest expression of MHC II and CD80 molecules on DCs was observed in the HES-MTX group, which confirms our previous observations concerning the modulatory potential of the nanoconjugate towards the DC phenotype. According to our assumptions, when immunotherapy was used, the high influx of Th, CTL and NKT cells into tumor nodules was accompanied by reduced infiltration of Tregs. It should be highlighted that the use of sole chemotherapy (i.e. MTX and HES-MTX groups of mice in the chemoimmunotherapeutic treatment scheme) was not sufficient to maintain the size of the tumor-infiltrating Treg population at a low level for a long time, as it was observed on the third day after administration of the chemotherapeutic treatment scheme. Regardless of a minor reduction in the $\mathrm{CD}^{+} \mathrm{T}$ cell percentage in the $\mathrm{MTX}+\mathrm{DC} / \mathrm{TAg}$ and HES-MTX+DC/TAg groups compared to the DC/TAg group, we observed higher percentage of the $\mathrm{NKT}$ and $\mathrm{CD}^{+} \mathrm{T}$ cells in those groups of mice; however, these differences were statistically significant only in the NKT cell population. Importantly, the highest percentage of $\mathrm{CD}^{+} \mathrm{T}$ cells was found in the HES-MTX-treated groups of mice, as in the previous experiment. At this point it is worth mentioning about the immunohistochemistry (IHC) techniques, which undoubtedly could provide the additional information about the localization of crucial immune cells in tumor nodules. However, due to the small size of the tumor tissue in some groups of mice and due to the planned extensive multi-parameter flow cytometric analyses, we decided to focus on the quantitative-flow cytometric assessment of immune cell infiltrating tumor tissue rather than qualitative determination of lymphocyte infiltration into tumor nodules (e.g. IHC). For this reason, we used the entire tumor tissue for flow cytometric analyses, which provided information about the percentage of immune cells present in the tumor in the context of other immune cell subpopulations, e.g. the percentage of $\mathrm{CD}^{+} \mathrm{T}$ cells among $\mathrm{CD} 45^{+}$ cells or percentage of Tregs among $\mathrm{CD} 4^{+} \mathrm{T}$ cells. Changes in the landscape of immune cells which occurred in tumor nodules were also reflected in the splenic lymphocyte populations. In the HES-MTX+DC/TAg group the percentages of splenic $\mathrm{CD}^{+}$and $\mathrm{CD} 8^{+}$cells were the lowest, while the percentage of Tregs were the highest. These changes may result from migration of activated splenic effector cells to the tumor growth site. Despite the fact that in the HES-MTX+DC/TAg group after restimulation the percentages of $\mathrm{CD}^{+}$and $\mathrm{CD} 49 \mathrm{~b}^{+}$cells were strongly reduced, the degranulation assay revealed the highest percentages of $\mathrm{CD}^{+}{ }^{+} \mathrm{CD} 107 \mathrm{a}^{+}, \mathrm{CD} 8{ }^{+} \mathrm{CD} 107 \mathrm{a}^{+}$and CD $49 \mathrm{~b}^{+} \mathrm{CD} 107 \mathrm{a}^{+}$. Despite the low percentage of $\mathrm{CD}_{4} 9 \mathrm{~b}^{+}$cells after restimulation, these cells possessed high cytotoxic potential, which was reflected in significant enhancement of cytotoxic activity of splenocytes in the HES-MTX+DC/TAg group. Similar observations were made by Zhong et al (53), who found that a combination of low-dose paclitaxel prior to intratumoral DC-based vaccine injection in a murine 3LL lung cancer model was more effective in inhibition of tumor growth. Moreover, combined therapy with paclitaxel and DC-based vaccines contributed to greater influx of $\mathrm{CD}^{+}$and $\mathrm{CD} 8^{+}$ $\mathrm{T}$ cells into tumor nodules and activation of a tumor-specific immune response in regional lymph nodes than either DC-vaccine or paclitaxel applied alone. The above results indicate that HES-MTX administration prior to DC-based immunotherapy affected the TME by eliminating certain cells with suppressor activity and increasing the influx of CTLs, which was beneficial for proper functioning of exogenous DCs. Moreover, this combined treatment had a positive impact on cytotoxic activity of splenic CTLs. Therefore, we postulate that activation of both the local and the systemic immune response finally contributed to inhibition of tumor growth.

In conclusion, our results show for the first time that the methotrexate nanoconjugate can modulate the systemic antitumor immune response and cause changes in the landscape of the TME through increasing influx of effector cells and eliminating certain cells with suppressor activity. Application of HES-MTX to MC38-tumor bearing mice resulted in induction of a systemic specific antitumor response. After therapy consisting of HES-MTX and DC-based vaccines CTL- and NK-mediated cytotoxicity was activated additionally. Moreover, in contrast to MTX application, HES-MTX used alone as well as together with DC-based cellular vaccines contributed to significant delay in tumor growth. However, only after treatment with HES-MTX and DC/TAg were there significant alterations noted in the proportions of crucial host immune cell populations necessary for an efficient antitumor response. Nevertheless, further research is required to determine which other factors present in the MC38 tumor microenvironment may have an adverse influence on functions of DCs administered as cellular vaccines and how we can overcome these difficulties.

\section{Acknowledgements}

Not applicable.

\section{Funding}

This research was supported by the National Science Centre, Poland (grant no. 2015/19/N/NZ6/02908 and 2017/27/B/NZ6/02702), and Wroclaw Centre of Biotechnology, The Leading National Research Centre (KNOW) program for the years 2014-2018. 


\section{Availability of data and materials}

The datasets used and/or analyzed during the current study are available from the corresponding author on reasonable request.

\section{Authors' contributions}

AS, JR and EPP conceptualized the research; JR, EPP and TMG developed the research methodology. AS and JR performed the formal analysis; AS, NAG, KWC, JM, TMG, MŚ, JR and EPP performed the experiments. AS and EPP wrote the manuscript. All the authors read and approved the manuscript and provided critical feedback and agree to be accountable for all aspects of the research in ensuring that the accuracy or integrity of any part of the work are appropriately investigated and resolved.

\section{Ethics approval and consent to participate}

All experiments were performed according to EU Directive 2010/63/EU for animal experiments and were approved by the 1st Local Ethics Committee for Experiments with the Use of Laboratory Animals, Wroclaw, Poland (number 31/2016).

\section{Patient consent for publication}

Not applicable.

\section{Competing interests}

The authors declare that they have no competing interests.

\section{References}

1. Nars MS and Kaneno R: Immunomodulatory effects of low dose chemotherapy and perspectives of its combination with immunotherapy. Int J Cancer 132: 2471-2478, 2013.

2. Golombek SK, May JN, Theek B, Appold L, Drude N, Kiessling F and Lammers T: Tumor targeting via EPR: Strategies to enhance patient responses. Adv Drug Deliv Rev 130: 17-38, 2018.

3. Roma-Rodrigues C, Mendes R, Baptista PV and Fernandes AR Targeting tumor microenvironment for cancer therapy. Int J Mol Sci 20: 840, 2019.

4. Hanahan D and Coussens LM: Accessories to the crime: Functions of cells recruited to the tumor microenvironment Cancer Cell 21: 309-322, 2012.

5. Whiteside TL: The tumor microenvironment and its role in promoting tumor growth. Oncogene 27: 5904-5912, 2008.

6. Jahchan NS, Mujal AM, Pollack JL, Binnewies M, Sriram V, Reyno L and Krummel MF: Tuning the tumor myeloid microenvironment to fight cancer. Front Immunol 10: 1611, 2019.

7. Netea-Maier RT, Smit JWA and Netea MG: Metabolic changes in tumor cells and tumor-associated macrophages: A mutual relationship. Cancer Lett 413: 102-109, 2018.

8. Veglia F and Gabrilovich DI: Dendritic cells in cancer: The role revisited. Curr Opin Immunol 45: 43-51, 2017.

9. Szczygieł A and Pajtasz-Piasecka E: Between biology and medicine: Perspectives on the use of dendritic cells in anticancer therapy. Postepy Hig Med Dosw (Online) 71: 921-941, 2017.

10. Constantino J, Gomes C, Falcão A, Cruz MT and Neves BM: Antitumor dendritic cell-based vaccines: Lessons from 20 years of clinical trials and future perspectives. Transl Res 168: 74-95, 2016.

11. Sabado RL, Balan S and Bhardwaj N: Dendritic cell-based immunotherapy. Cell Res 27: 74-95, 2017.

12. Palucka K and Banchereau J: Dendritic-cell-based therapeutic cancer vaccines. Immunity 39: 38-48, 2013.
13. Bol KF, Schreibelt G, Gerritsen WR, de Vries IJM and Figdor CG: Dendritic cell-based immunotherapy: State of the art and beyond. Clin Cancer Res 22: 1897-1906, 2016.

14. Goszczyński T, Nevozhay D, Wietrzyk J, Omar MS and Boratyński J: The antileukemic activity of modified fibrinogen-methotrexate conjugate. Biochim Biophys Acta 1830: 2526-2530, 2013.

15. Hao F, Lee RJ, Yang C, Zhong L, Sun Y, Dong S, Cheng Z, Teng L, Meng Q, Lu J, et al: Targeted Co-delivery of siRNA and methotrexate for tumor therapy via mixed micelles. Pharmaceutics 11: 92, 2019.

16. Chen Y,Zhang W, Huang Y, Gao F, Sha X, Lou K and Fang X: The therapeutic effect of methotrexate-conjugated Pluronic-based polymeric micelles on the folate receptor-rich tumors treatment. Int J Nanomedicine 10: 4043-4057, 2015.

17. Thomas TP, Huang B, Choi SK, Silpe JE, Kotlyar A, Desai AM, Zong H, Gam J, Joice $M$ and Baker JR Jr: Polyvalent dendrimer-methotrexate as a folate receptor-targeted cancer therapeutic. Mol Pharm 9: 2669-2676, 2012.

18. Li MH, Choi SK, Thomas TP, Desai A, Lee KH, Kotlyar A, Banaszak Holl MM and Baker JR Jr: Dendrimer-based multivalent methotrexates as dual acting nanoconjugates for cancer cell targeting. Eur J Med Chem 47: 560-572, 2012.

19. Figueiró F, de Oliveira CP, Bergamin LS, Rockenbach L, Mendes FB, Jandrey EH, Moritz CE, Pettenuzzo LF, Sévigny J, Guterres SS, et al: Methotrexate up-regulates ecto-5'-nucleotidase/CD73 and reduces the frequency of T lymphocytes in the glioblastoma microenvironment. Purinergic Signal 12: 303-312, 2016.

20. Goszczyński TM, Filip-Psurska B, Kempińska K, Wietrzyk J and Boratyński J: Hydroxyethyl starch as an effective methotrexate carrier in anticancer therapy. Pharmacol Res Perspect 2: e00047, 2014.

21. Goszczyński T, Boratyński J, Wietrzyk J, Filip-Psurska B and Kempińska K: Patent WO/2013/127885; A conjugate of methotrexate and Hydroxyethyl starch for use in the treatment cancer, 2013.

22. Koźmiński P, Halik PK, Chesori R and Gniazdowska E: Overview of dual-acting drug methotrexate in different neurological diseases, autoimmune pathologies and cancers. Int J Mol Sci 21: 3483, 2020.

23. Abolmaali SS, Tamaddon AM and Dinarvand R: A review of therapeutic challenges and achievements of methotrexate delivery systems for treatment of cancer and rheumatoid arthritis. Cancer Chemother Pharmacol 71: 1115-1130, 2013.

24. Visentin M, Zhao R and Goldman ID: The antifolates. Hematol Oncol Clin North Am 26: 629-648, ix, 2012.

25. Brecher ME, Owen HG and Bandarenko N: Alternatives to albumin: Starch replacement for plasma exchange. J Clin Apher 12: 146-153, 1997.

26. Hoffmann S, Caysa H, Kuntsche J, Kreideweiss P, Leimert A, Mueller T and Mäder K: Carbohydrate plasma expanders for passive tumor targeting: In vitro and in vivo studies. Carbohydr Polym 95: 404-413, 2013.

27. Nogueira E, Sárria MP, Azoia NG, Antunes E, Loureiro A, Guimarães D, Noro J, Rollett A, Guebitz G and Cavaco-Paulo A: Internalization of methotrexate conjugates by folate receptor- $\alpha$. Biochemistry 57: 6780-6786, 2018.

28. Thomas TP, Goonewardena SN, Majoros IJ, Kotlyar A, Cao Z, Leroueil PR and Baker JR: Folate-targeted nanoparticles show efficacy in the treatment of inflammatory arthritis. Arthritis Rheum 63: 2671-2680, 2011.

29. Wang S and Dormidontova EE: Nanoparticle targeting using multivalent ligands: Computer modeling. Soft Matter 7: 4435-4445, 2011

30. Fang J, Nakamura $H$ and Maeda H: The EPR effect: Unique features of tumor blood vessels for drug delivery, factors involved, and limitations and augmentation of the effect. Adv Drug Deliv Rev 63: 136-151, 2011.

31. Maeda H: Tumor-selective delivery of macromolecular drugs via the EPR effect: Background and future prospects. Bioconjug Chem 21: 797-802, 2010.

32. Maeda $\mathrm{H}$ and Matsumura Y: EPR effect based drug design and clinical outlook for enhanced cancer chemotherapy. Adv Drug Deliv Rev 63: 129-130, 2011.

33. Shurin GV, Tourkova IL, Kaneno R and Shurin MR: Chemotherapeutic agents in noncytotoxic concentrations increase antigen presentation by dendritic cells via an IL-12-dependent mechanism. J Immunol 183: 137-144, 2009. 
34. Kaneno R, Shurin GV, Tourkova IL and Shurin MR: Chemomodulation of human dendritic cell function by antineoplastic agents in low noncytotoxic concentrations. J Transl Med 7: 58 2009.

35. Kaneno R, Shurin GV, Kaneno FM, Naiditch H, Luo J and Shurin MR: Chemotherapeutic agents in low noncytotoxic concentrations increase immunogenicity of human colon cancer cells. Cell Oncol (Dordr) 34: 97-106, 2011.

36. Zhang L, Dermawan K, Jin M, Liu R, Zheng H, Xu L, Zhang Y, Cai Y, Chu Y and Xiong S: Differential impairment of regulatory $\mathrm{T}$ cells rather than effector $\mathrm{T}$ cells by paclitaxel-based chemotherapy. Clin Immunol 129: 219-229, 2008.

37. Sistigu A, Viaud S, Chaput N, Bracci L, Proietti E and Zitvogel L: Immunomodulatory effects of cyclophosphamide and implementations for vaccine design. Semin Immunopathol 33: 369-383, 2011.

38. Liu JY, Wu Y, Zhang XS, Yang JL, Li HL, Mao YQ, Wang Y, Cheng X, Li YQ, Xia JC, et al: Single administration of low dose cyclophosphamide augments the antitumor effect of dendritic cell vaccine. Cancer Immunol Immunother 56: 1597-1604, 2007.

39. Rossowska J, Anger N, Kicielińska J, Pajtasz-Piasecka E, Bielawska-Pohl A, Wojas-Turek J and Duś D: Temporary elimination of IL-10 enhanced the effectiveness of cyclophosphamide and BMDC-based therapy by decrease of the suppressor activity of MDSCs and activation of antitumour immune response. Immunobiology 220: 389-398, 2015.

40. Rossowska J, Pajtasz-Piasecka E, Anger N, Wojas-Turek J, Kicielińska J, Piasecki E and Duś D: Cyclophosphamide and IL-12-transduced DCs enhance the antitumor activity of tumor antigen-stimulated DCs and reduce Tregs and MDSCs number J Immunother 37: 427-439, 2014.

41. Pajtasz-Piasecka E, Szyda A, Rossowska J, Krawczenko A, Indrová M, Grabarczyk P, Wysocki P, Mackiewicz A and Duś D: Loss of tumorigenicity of murine colon carcinoma MC38/0 cell line after transduction with a retroviral vector carrying murine IL-12 genes. Folia Biol (Praha) 50: 7-14, 2004

42. Rossowska J, Pajtasz-Piasecka E, Szyda A, Krawczenko A, Zietara N and Dus D: Tumour antigen-loaded mouse dendritic cells maturing in the presence of inflammatory cytokines are potent activators of immune response in vitro but not in vivo. Oncol Rep 21: 1539-1549, 2009.

43. Thomas E, Jones G, de Souza P, Wardrop C and Wusteman F: Measuring blood volume with fluorescent-labeled hydroxyethyl starch. Crit Care Med 28: 627-631, 2000.

44. Nevozhay D, Budzynska R, Kanska U, Jagiello M, Omar MS, Boratynski J and Opolski A: Antitumor properties and toxicity of dextran-methotrexate conjugates are dependent on the molecular weight of the carrier. Anticancer Res 26: 1135-1143, 2006.
45. Ciekot J, Goszczyński T and Boratyńskit J: Methods for methotrexate determination in macromolecular conjugates drug carrier. Acta Pol Pharm 69: 1342-1346, 2012.

46. Rossowska J, Anger N, Szczygieł A, Mierzejewska J and Pajtasz-Piasecka E: Reprogramming the murine colon cancer microenvironment using lentivectors encoding shRNA against IL-10 as a component of a potent DC-based chemoimmunotherapy. J Exp Clin Cancer Res 37: 126, 2018.

47. Pajtasz-Piasecka E, Rossowska J, Szyda A, Krawczenko A and Dus D: Generation of anti-tumor response by JAWS II mouse dendritic cells transduced with murine interleukin 12 genes. Oncol Rep 17: 1249-1257, 2007.

48. Ji X, Guo H, Tang Q, Ma D and Xue W: A targeted nanocarrier based on polyspermine for the effective delivery of methotrexate in nasopharyngeal carcinoma. Mater Sci Eng C Mater Biol Appl 81: 48-56, 2017.

49. Goos JACM, Cho A, Carter LM, Dilling TR, Davydova M, Mandleywala K, Puttick S, Gupta A, Price WS, Quinn JF, et al: Delivery of polymeric nanostars for molecular imaging and endoradiotherapy through the enhanced permeability and retention (EPR) effect. Theranostics 10: 567-584, 2020.

50. Kobayashi H, Watanabe R and Choyke PL: Improving conventional enhanced permeability and retention (EPR) effects; what is the appropriate target? Theranostics 4: 81-89, 2013.

51. Pajtasz-Piasecka E and Indrová M: Dendritic cell-based vaccines for the therapy of experimental tumors. Immunotherapy 2 : 257-268, 2010.

52. Zong J, Keskinov AA, Shurin GV and Shurin MR: Tumor-derived factors modulating dendritic cell function. Cancer Immunol Immunother 65: 821-833, 2016.

53. Zhong H, Han B, Tourkova IL, Lokshin A, Rosenbloom A, Shurin MR and Shurin GV: Low-dose paclitaxel prior to intratumoral dendritic cell vaccine modulates intratumoral cytokine network and lung cancer growth. Clin Cancer Res 13: 5455-5462, 2007.

54. Rossowska J, Anger N, Szczygieł A, Mierzejewska J and Pajtasz-Piasecka E: Intratumoral lentivector-mediated TGF- $\beta 1$ gene downregulation as a potent strategy for enhancing the antitumor effect of therapy composed of cyclophosphamide and dendritic cells. Front Immunol 8: 713, 2017.

This work is licensed under a Creative Commons Attribution-NonCommercial 4.0 International (CC BY-NC 4.0) License. 